\title{
Regioselective synthesis of substituted piperidine-2,4-diones and their derivatives via Dieckmann cyclisations
}

\author{
Charles M. Marson* and Kin Cheung Yau
}

Department of Chemistry, University College London, Christopher Ingold Laboratories, 20 Gordon Street, London WC1H OAJ, U.K.

\begin{abstract}
A flexible route to piperidine-2,4-diones variously substituted at the 6-, 5,6- and 2,6-positions, both with and without 1-substitution, is described; no $\mathrm{N}$-protective group is required. A related regioselective Dieckmann cyclisation is also described that uses Davies' $\alpha$-methylbenzylamine auxiliary and affords 6-substituted piperidine-2,4-diones enantioselectively.
\end{abstract}

Keywords: Dieckmann cyclisation, piperidine-2,4-diones, regioselective synthesis, enantioselective synthesis

\section{Introduction}

The piperidine ring, a privileged scaffold in pharmaceutically active compounds, ${ }^{1}$ is exemplified in the numerous piperidine alkaloids. ${ }^{2,3}$ Oxygenated forms of the piperidine ring such as substituted piperidin-2-ones are also present in a number of alkaloids ${ }^{3}$ and in other biologically active derivatives, ${ }^{4}$ and can confer advantages of increased stability and crystallinity compared with piperidine analogues. Diversely substituted piperidines and oxygenated piperidines find much use in drug development, but their synthesis is still challenging, ${ }^{5}$ general, scaleable methods are in demand. Many oxopiperidines including piperidine-2,4-diones possess biological and pharmaceutical relevance (Fig. 1), ${ }^{6,7}$ some being key intermediates in the synthesis of kinase inhibitors ${ }^{6 \mathrm{f}}$ and modulators of glutamate receptors ${ }^{6 g}$ but routes to 6-substituted piperidine-2,4-diones are limited. ${ }^{8}$ Enantiocontrolled strategies to substituted piperidines present a further challenge, although the work of Comins ${ }^{9}$ and Davis ${ }^{10}$ has addressed some of the limitations in the range or location of substituents. Enantioselective syntheses of 1-unsubstituted piperidine-2,4-diones include rearrangements of substituted 1,3 -oxazinan-2-ones, ${ }^{8}$ and cyclisations of $N$-sulfinyl $\delta$-amino- $\beta$-keto esters, ${ }^{10} \delta$ aryl- $\delta$-amino- $\beta$-keto esters ${ }^{6 \mathrm{~d}}$ and $N$-Boc- $\beta$-amino acids. ${ }^{11}$ Herein, we describe the synthesis of variously substituted piperidine-2,4-diones prepared via a regioselective Dieckmann cyclisation, some being obtained enantioselectively.

Corresponding author's e-mail address: c.m.marson@ucl.ac.uk (C. M. Marson) 


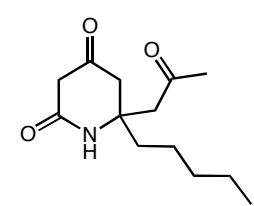

Adalinine $e^{3 b}$<smiles>[R]C=C(C)C(=O)C1=C(O)CCNC1=O</smiles>

Subunit of kirromycin antibiotics ${ }^{7 a}$<smiles>COc1ccc(C2CC(OCC(C)C)=CC(=O)N2)cc1OC</smiles>

Anti-hypoglycaemic agent ${ }^{6 c}$

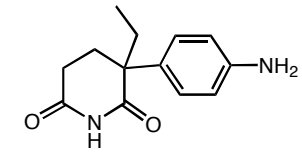

Aminoglutethimide, aromatase inhibitor $^{7 \mathrm{~b}}$<smiles>O=C1CC2(CCCC2)C(=O)N1</smiles>

$\alpha_{1 d}$ Adrenergic receptor antagonists $^{7 c}$

Figure 1. Representative oxopiperidine derivatives with biological or pharmaceutical activity.

\section{Results and Discussion}

It was desired to investigate the scope of Dieckmann cyclisations for the synthesis of piperidine-2,4-diones in regard to substituent location, and in particular to achieve a succinct protocol for the preparation of $\mathrm{N}$-unsubstituted piperidine-2,4-diones, since few such Dieckmann cyclisations have been described, and a cyclisation in THF that afforded 3methoxycarbonyl-6-(2-phenethyl)piperidine-2,4-dione in $15 \%$ yield. ${ }^{12}$ The proposed route (Scheme 1) began with $\beta$-keto esters $\mathbf{1}$, which in the cases of $\mathbf{1 b}$ and $\mathbf{1 c}$ were prepared by Weiler alkylation ${ }^{13}$ of the dianion of methyl acetoacetate (1a), prepared in THF using NaH (1.1 equiv.) and butyllithium (1.1 equiv.), and reacted with methyl iodide or ethyl bromide, respectively (Scheme 2). Subsequent alkylation at the $\beta$-position was also possible, ester 1b being converted into 1d. Reaction of $\beta$-keto esters 1 with ammonium acetate in the presence of acetic acid afforded the vinylogous carbamates $\mathbf{2}$ which were reduced with sodium triacetoxyborohydride prepared in situ ${ }^{14}$ to give the corresponding $\beta$-amino esters 3 . The latter were coupled with monomethyl malonate using EDC in the presence of $\mathrm{HOBt}$ to give the amidodiesters 4. Those underwent Dieckmann cyclisation upon treatment with sodium methoxide in methanol to give the keto esters $\mathbf{5}$ which were hydrolysed ( $\mathrm{NaOMe}$ in methanol) and decarboxylated using sodium methoxide in wet acetonitrile ${ }^{6 \mathrm{~b}}$ in a one-pot process to give the corresponding piperidine-2,4-diones 6.

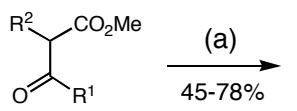

1<smiles>[R]C(NC(=O)CC(C)=O)C([R])C(C)=O</smiles>

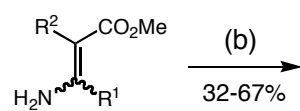

2<smiles>[R]C1NC(=O)C(C(C)=O)=C(O)C1[R]</smiles><smiles>[R]C(N)C([R])C(C)=O</smiles>

3

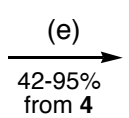<smiles>[R]C1NC(=O)CC(=O)C1[R]</smiles>

6 a $\mathrm{R}^{1}=\mathrm{Me}, \mathrm{R}^{2}=\mathrm{H}$

b $R^{1}=E t, R^{2}=H$

c $\mathrm{R}^{1}=n-\operatorname{Pr}, \mathrm{R}^{2}=\mathrm{H}$

d $R^{1}=E t, R^{2}=M e$

e $\mathrm{R}^{1}=\mathrm{CH}_{2} \mathrm{Ph}, \mathrm{R}^{2}=\mathrm{Me}$

f $\mathrm{R}^{1}=\mathrm{Ph}, \mathrm{R}^{2}=\mathrm{H}$

g R $\mathrm{R}^{1}=3-$ Pyridyl, $\mathrm{R}^{2}=\mathrm{H}$

Scheme 1. Synthesis of substituted piperidine-2,4-diones and derivatives via Dieckmann cyclisations. Reagents and conditions: (a) $\mathrm{NH}_{4} \mathrm{OAc}, \mathrm{AcOH}, \mathrm{PhH}$, reflux, 18-72 h; (b) $\mathrm{NaBH}_{4}$ (2.5 equiv.) AcOH, 20 
${ }^{\circ} \mathrm{C}, 3 \mathrm{~h}$; (c) monomethyl malonate, EDC (1 equiv.), $\mathrm{HOBt}\left(1.5\right.$ equiv.), $\left(i\right.$-Pr) ${ }_{2} \mathrm{NEt}, \mathrm{CH}_{2} \mathrm{Cl}_{2}, 20{ }^{\circ} \mathrm{C}, 2 \mathrm{~h}$; (d) $\mathrm{NaOMe}$ (2 equiv.), $\mathrm{MeOH}$, reflux, $1 \mathrm{~h}$; (e) $\mathrm{MeCN}, 1 \% \mathrm{H}_{2} \mathrm{O}$, reflux, $1 \mathrm{~h}$.

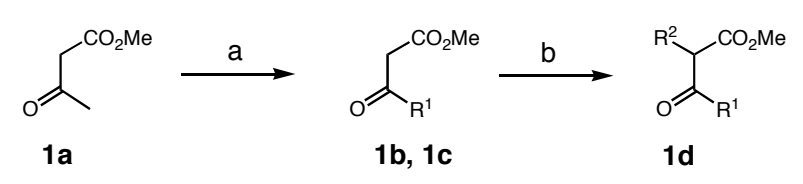

Scheme 2. Synthesis of $\beta$-keto esters via Weiler dialkylation. Reagents and conditions: (a) NaH (1.1 equiv.), BuLi (1.1 equiv.), THF, MeI or EtBr (1.1equiv.) EtOH, $20{ }^{\circ} \mathrm{C}, 3 \mathrm{~h}, 97 \%$ (1b), 85\%, (1c); (b) MeI (1.0 equiv.), $\mathrm{K}_{2} \mathrm{CO}_{3}$ (1.5 equiv.), $\mathrm{Me}_{2} \mathrm{CO}, 0{ }^{\circ} \mathrm{C}, 2 \mathrm{~h}, 88 \%$ (1d).

For the 2,3-disubstitued $\beta$-amino esters 3, a Blaise reaction ${ }^{15}$ (Scheme 3) with in situ reduction was investigated, and afforded 6e; however, the poor yield suggested that the reductive amination of $\beta$-keto esters $\mathbf{1}$, as in Scheme 1, would in general be preferable.

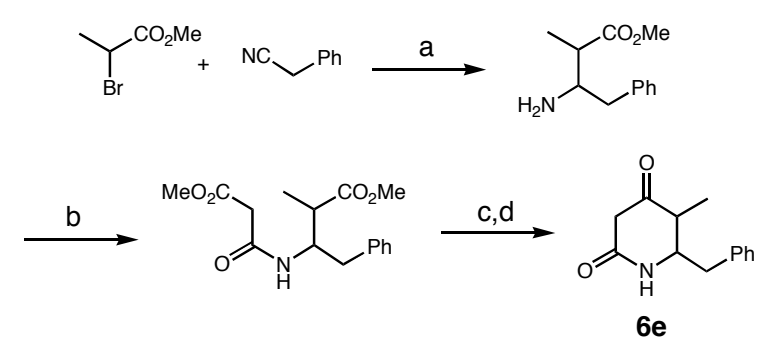

Scheme 3. Synthesis of 2,3-disubstituted piperidine-2,4-diones. Reagents and conditions: (a) Zn (4 equiv.), TMSCl (12 equiv.), $\mathrm{CH}_{2} \mathrm{Cl}_{2}$, THF, reflux, $2 \mathrm{~h}$ then $\mathrm{NaBH}_{4}\left(0.55\right.$ mole equiv.) EtOH, $20^{\circ} \mathrm{C}, 3 \mathrm{~h}$, $15 \%$; (b) monomethyl malonate, EDC (1.0 equiv.), $\mathrm{HOBt}\left(1.5\right.$ equiv.), $(i \text { - } \mathrm{Pr})_{2} \mathrm{NEt}, \mathrm{CH}_{2} \mathrm{Cl}_{2}, 20{ }^{\circ} \mathrm{C}, 2 \mathrm{~h}$, $67 \%$; (c) $\mathrm{NaOMe}$ (1.3 equiv.), $\mathrm{MeOH}$, reflux, $1 \mathrm{~h}$, then $\mathrm{MeCN}, 1 \% \mathrm{H}_{2} \mathrm{O}$, reflux, $1 \mathrm{~h}, 56 \%$.

These results show that a variety of 6-monosubstituted piperidine-2,4-diones can be prepared by the protocol of Scheme 1, and that in all of the examples studied cyclisation proceeded satisfactorily and usually in good yields. 5,6-Disubstituted piperidine-2,4-diones can also be prepared, although not with high diastereoselectivity in the examples studied herein. Alkylation of $\mathbf{6 f}$ with $\mathrm{MeI}$ ( 3 equiv.) in acetone at $50{ }^{\circ} \mathrm{C}$ in the presence of potassium carbonate afforded the 3,3,6-trisubstituted piperidine-2,4-dione $\mathbf{6 h}(65 \%)$; no monomethyl product could be isolated, even when 1 equiv. of MeI was used. Accordingly, monomethylation of ester $\mathbf{5 f}$ was attempted using the conditions above, but no 6i was observed; however, using Page's procedure ${ }^{16}$ alkylation with MeI (2 equiv.) in THF at $20{ }^{\circ} \mathrm{C}$ was achieved, affording $6 \mathbf{i}$ in $64 \%$ yield. Attempts to remove the methoxycarbonyl group from $\mathbf{6 i}$, including sodium chloride in DMSO,${ }^{17} \mathrm{LiI}$ in pyridine, wet acetonitrile, or hydrochloric acid $(1.3 \mathrm{M})$, all at reflux, were not successful. 
<smiles>CC(=O)C1=C(O)CC(c2ccccc2)NC1=O</smiles><smiles>CC1C(=O)CC(=O)NC1Cc1ccccc1</smiles><smiles>CC1CC(=O)CC(=O)N1</smiles>

6a<smiles>O=C1CC(=O)NC(c2ccccc2)C1</smiles><smiles>CCCC1CC(=O)CC(=O)N1</smiles>

6c<smiles>CCC1NC(=O)CC(=O)C1C</smiles>

$6 d$

Figure 2. Synthesis of substituted piperidine-2,4-diones and derivatives via Dieckmann cyclisations.

The scope of the 2-substituent appears to include 3-pyridyl, since the formation of $\mathbf{6 g}$ (Scheme 4) was confirmed by ${ }^{1} \mathrm{H}$ NMR spectroscopy, but the aqueous solubility of this piperidine-2,4-dione precluded isolation under the standard procedures attempted.

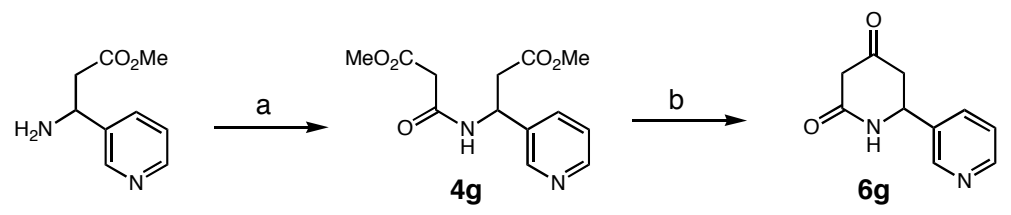

Scheme 4. Attempted cyclisation of diester 4g. Reagents and conditions: (a) $\mathrm{ClOCCH}_{2} \mathrm{CO}_{2} \mathrm{Me}(1.5$ equiv.), ( $i$-Pr) $)_{2} \mathrm{NEt}$ (4 equiv.), $0{ }^{\circ} \mathrm{C}$, then $20^{\circ} \mathrm{C}, 1 \mathrm{~h}, 99 \%$; (b) $\mathrm{NaOMe}$ (1.3 equiv.), $\mathrm{MeOH}$, reflux, $1 \mathrm{~h}$, then $\mathrm{MeCN}, 1 \% \mathrm{H}_{2} \mathrm{O}$, reflux, $1 \mathrm{~h}$.

The present study has established a greater scope of Dieckmann cyclisations that afford substituted piperidine-2,4-diones (Fig. 2), notably $N$-unsubstituted compounds; the use of dimethyl ester precursors, cyclised in methanol (in the presence of sodium methoxide), followed by decarbomethoxylation in aqueous acetonitrile appears to be an improved procedure for obtaining such Dieckmann products; for example, using those procedures, cyclisation and decarbomethoxylation afforded 6-phenylpiperidine-2,4-dione (6f) in 66\% yield, compared with $32 \%$ using previous conditions. ${ }^{6 \mathrm{~b}}$ Convenient features of this synthetic approach include the ready preparation of a wide variety of $\beta$-amino esters from the corresponding $\beta$-keto esters, and the avoidance of $N$-protection followed by $N$-deprotection; the substituted piperidine-2,4-diones are obtained directly as $\mathrm{N}$-unsubstituted compounds, as are all intermediates. The route is compatible with one or more substituents at the 3-, 5- and 6-positions.

An enantioselective route to substituted piperidine-2,4-diones based on the regioselective Dieckmann cyclisation was then investigated (Scheme 5). The enantiomerically pure $\beta$-keto esters 8 were prepared by metalation of 7 and reaction with $\alpha, \beta$-unsaturated esters, according to the Davies methodology. ${ }^{18}$ Deallylation of allylamines 8 was achieved using Wilkinson's catalyst $^{186,19}$ via isomerisation to the corresponding enamides that are hydrolysed in situ. ${ }^{20}$ 
However, in the case of $\mathbf{8 g}$ several products were obtained; accordingly, allylic transfer was investigated, and was achieved using 1,3-dimethylbarbituric acid (3 equiv.) with a catalytic quantity of $\left(\mathrm{Ph}_{3} \mathrm{P}\right)_{4} \mathrm{Pd}$ in dichloromethane, affording $9 \mathrm{~g}$ in $96 \%$ yield. Acylation of $\beta$-amino esters 9 using monomethyl malonate and EDC or, where that proved unsatisfactory, methyl malonyl chloride and $\mathrm{Et}_{3} \mathrm{~N}$ afforded the corresponding malonamides $\mathbf{1 0}$ which underwent ring closure upon treatment with sodium methoxide in ethanol; in two cases the intermediate sodium salts $\mathbf{1 1}$ were isolated, but since those were only needed to confirm the course of the reaction, direct ester hydrolysis and decarboxylation was otherwise achieved, either using warm dilute hydrochloric acid at reflux or in wet acetonitrile at reflux, affording the enantiopure 6-piperidine-2,4-diones $\mathbf{1 2}$ in a one-pot procedure from diesters $\mathbf{1 0}$. Using methanesulfonic acid (0.9 equiv.) in toluene at reflux, ${ }^{21}$ several of the $\mathrm{N}$-alkylated products $\mathbf{1 2}$ were cleaved to the corresponding enantiopure piperidine-2,4-diones 6 lacking a 1-substituent (Fig. 3). 3,3-Dimethylation of $\mathbf{6 1}$ with methyl iodide (3 equiv.) in methanol in the presence of potassium carbonate afforded the 3,3,6-trisubstituted piperidine-2,4-dione 13. The route can also be used to prepare enantiomerically pure 4-hydroxypiperidinones, as illustrated by the reduction of $(R)$-6f to $\mathbf{1 4}$ using zinc borohydride. ${ }^{22}$ The 3-pyridyl derivative $\mathbf{1 2 f}$ could be a useful building block for the enantioselective synthesis of novel substituted piperidines related to the alkaloid anabasine.

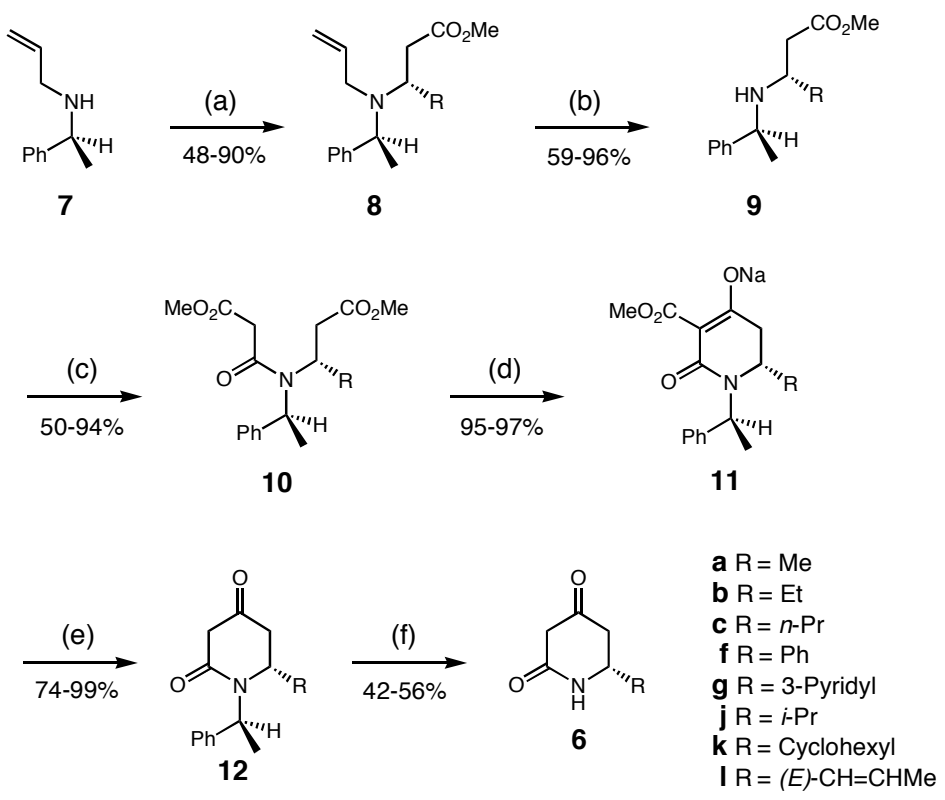

Scheme 5. Enantioselective synthesis of substituted piperidine-2,4-diones and derivatives via Dieckmann cyclisations $(\mathrm{R}=(S)-1$-phenylethyl). Reagents and conditions: (a) BuLi (1 equiv.), THF, $78{ }^{\circ} \mathrm{C}, 30 \mathrm{~min}$; $\mathrm{RCH}=\mathrm{CHCO}_{2} \mathrm{Me}(1$ equiv. $),-78{ }^{\circ} \mathrm{C}, 30 \mathrm{~min}$; (b) $\left(\mathrm{Ph}_{3} \mathrm{P}\right)_{3} \mathrm{RhCl}(5 \mathrm{~mol} \%)$, aq. $\mathrm{MeCN}$, reflux, $16 \mathrm{~h}$; (c) methyl 3-chloro-3-oxopropanoate (1.1 equiv.), $\mathrm{Et}_{3} \mathrm{~N}$ (1.2 equiv.), $0{ }^{\circ} \mathrm{C}$ to $20{ }^{\circ} \mathrm{C}, 1 \mathrm{~h}$; (d) $\mathrm{NaOMe}(1.1$ equiv.), reflux $1 \mathrm{~h}$, then dil. $\mathrm{HCl}$; (e) $1 \%$ water in $\mathrm{MeCN}$, reflux $1 \mathrm{~h}$; (f) $\mathrm{MsOH}(0.9$ equiv.), toluene, reflux, $3 \mathrm{~h}$. 


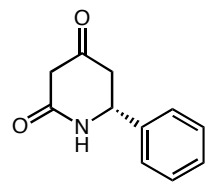

(R)-6f<smiles>CC(C)[C@H]1CC(=O)CC(=O)N1</smiles>

$(S)-6 \mathbf{j}$<smiles>O=C1CC(=O)N[C@H](C2CCCCC2)C1</smiles>

(R)-6k
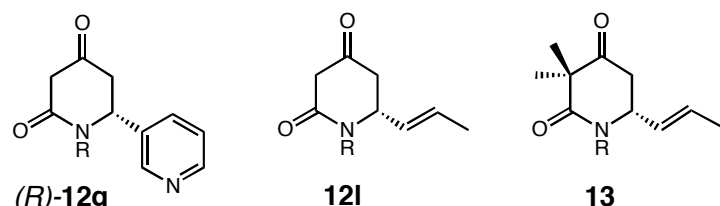

13

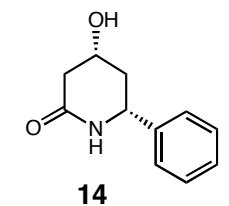

14

Figure 3. Enantioselective synthesis of substituted piperidine-2,4-diones and derivatives via Dieckmann cyclisations $(\mathrm{R}=(S)-1$-phenylethyl).

This study shows that a variety of substituted piperidine-2,4-diones, in which 1-substitution may be present or absent, can be conveniently prepared by Dieckmann cyclisation. Use of the Davies $^{18}$ conjugate addition of ( $\left.S\right)-N$-( $\alpha$-methylbenzyl)allylamine (7) to $\alpha, \beta$-unsaturated esters afforded enantiopure $\beta$-amino esters that also underwent Dieckmann cyclisation to give, after hydrolysis and decarboxylation, the corresponding substituted piperidine-2,4-diones; the chiral auxiliary was cleaved using methanesulfonic acid, thereby achieving some enantioselective syntheses of substituted piperidine-2,4-diones without 1-substitution. Synthesis of substituted piperidine-2,4-diones can be used to access to a variety of congeners of alkaloids or other pharmacologically active compounds, including 4-hydroxypiperidin-2-ones, the corresponding substituted piperidin-2-ones and piperidines; examples herein include the piperidine-2,4-dione $\mathbf{1 2 g}$, an analogue of anabasine, and $(4 R, 6 R)$-4-hydroxy-6-phenylpiperidin-2-one (14) prepared by reduction of $\mathbf{6 f}$ with $\mathrm{LiAlH}_{4}$.

\section{Experimental Section}

3.1 General. All moisture-sensitive reactions were performed under a nitrogen atmosphere and the glassware was pre-dried in an oven $\left(130^{\circ} \mathrm{C}\right)$. Evaporation refers to the removal of solvent under reduced pressure. Melting points were measured by a microscope hot-stage Electrothermal 9100 apparatus. Infra-red (IR) spectra were recorded on a Perkin-Elmer PE-983 spectrophotometer; absorptions are quoted in wavenumbers. ${ }^{1} \mathrm{H}$ NMR spectra were recorded on a Bruker AC300 (300 MHz) spectrometer or a Bruker AMX 500 (125 MHz) spectrometer; data are reported in parts per million $(\delta)$. Coupling constants $(J)$ are given in Hertz $(\mathrm{Hz})$. The following abbreviations were used in signal assignments: singlet (s), broad singlet (br s), doublet $(\mathrm{d})$, triplet $(\mathrm{t})$, quartet $(\mathrm{q})$, and multiplet $(\mathrm{m}) .{ }^{13} \mathrm{C}$ NMR spectra were recorded on a Bruker AC300 (300 MHz) spectrometer or a Bruker AMX $500(125 \mathrm{MHz})$ spectrometer; data are reported in parts per million (d), with $\mathrm{CHCl}_{3}$ as an internal standard. Mass spectra were recorded on a VG7070H mass spectrometer with Finigan Incos II data system at University College London. Optical rotations were measured using a Perkin-Elmer 343 digital polarimeter. 
Thin-layer chromatography was performed on Merck $0.2 \mathrm{~mm}$ aluminium-backed silica gel 60 $\mathrm{F}_{254}$ plates and visualised by $\mathrm{UV}(254 \mathrm{~nm})$ or by staining with potassium permanganate with subsequent heating. Flash column chromatography was performed using Merck 0.040-0.063 $\mathrm{mm}, 230-400$ mesh silica gel. Temperatures below $0{ }^{\circ} \mathrm{C}$ were obtained using various mixtures of water, salt and ice, or acetone and dry ice.

The following compounds were prepared according to the literature: methyl 2-methyl-3oxopentanoate (1d); ${ }^{23}$ methyl 3-amino-3-phenylpropanoate hydrochloride $(\mathbf{3 f}) ;{ }^{24}$ methyl 3-(2methoxycarbonylacetylamino)-3-phenylpropanoate $(\mathbf{4 f}){ }^{25}(S)-N$-( $\alpha$-methylbenzyl)allylamine (7) $;{ }^{26}(E)$-methyl 4-methylpent-2-enoate ${ }^{27}(E, E)$-methyl hexa-2,4-dienoate ${ }^{28}$ methyl $(2 E)$-3cyclohexyl-2-propenoate, ${ }^{29}(E)$-methyl 3-(pyridin-3-yl)propenoate. ${ }^{30}$

General procedure A. Preparation of amidodiesters 4 and 10. Anhydrous 1hydroxybenzotriazole (1.5 equiv.) and $N, N$-diisopropylethylamine (4.0 equiv.) were added to a stirred solution of the $\beta$-amino ester 3 (1.0 equiv.) in dichloromethane at $0{ }^{\circ} \mathrm{C}$, under nitrogen. Monomethyl malonate (3.0 equiv.) in dichloromethane was added dropwise; $\mathrm{N}$-(3dimethylaminopropyl)- $N$ '-ethylcarbodiimide hydrochloride (1.0 equiv.) was added and the mixture was then allowed to warm to $20{ }^{\circ} \mathrm{C}$ and stirred for $2 \mathrm{~h}$. Saturated aqueous sodium hydrogen carbonate was then added and the aqueous layer was extracted with ethyl acetate and the combined organic layers were washed with brine, dried $\left(\mathrm{MgSO}_{4}\right)$ and evaporated. The residue was purified as described under the given product.

General procedure B. Preparation of amido diesters 4. Triethylamine (1.3 equiv.) and methyl 3-chloro-3-oxopropanoate (1.2 equiv.) were added to a stirred solution of the $\beta$-amino ester 3 ( 1 equiv.) in dichloromethane at $0{ }^{\circ} \mathrm{C}$, under nitrogen. The mixture was then allowed to warm to $20^{\circ} \mathrm{C}$ and stirred for $1 \mathrm{~h}$. The mixture was diluted with dichloromethane and washed with saturated aqueous sodium hydrogen carbonate. The aqueous layer was extracted twice with dichloromethane and the combined organic layers were dried $\left(\mathrm{MgSO}_{4}\right)$ and evaporated. The residue was purified as described under the given product.

General procedure C for Dieckmann cyclisations. Sodium methoxide (1.2 equiv.) in methanol was added to a stirred solution of the diester (1.0 equiv.) in methanol at $20{ }^{\circ} \mathrm{C}$ under nitrogen. The mixture was then heated under reflux for $1 \mathrm{~h}$. After allowing to cool to $20^{\circ} \mathrm{C}$, the mixture was acidified with hydrochloric acid $(1 \mathrm{M})$ to $\mathrm{pH}$ 6. The aqueous layer was extracted with dichloromethane, dried $\left(\mathrm{MgSO}_{4}\right)$, and evaporated. A solution of $1 \%$ water in acetonitrile was added to the oily residue, and the mixture was heated under reflux for $1 \mathrm{~h}$. 
The mixture was allowed to cool, then evaporated, and the residue was purified as described under the given product.

General procedure D for Michael addition. ${ }^{18} n$-Butyllithium (1.55 equiv.) in THF was added dropwise via a syringe to a stirred solution of $(S)-N$-( $\alpha$-methylbenzyl)allylamine (7) (1.6 equiv.) in anhydrous THF at $-78{ }^{\circ} \mathrm{C}$ under nitrogen. The mixture was stirred at $-78{ }^{\circ} \mathrm{C}$ for a further $30 \mathrm{~min}$. A solution of the $\alpha, \beta$-unsaturated ester (1.0 equiv.) in anhydrous THF was added dropwise via syringe at $-78^{\circ} \mathrm{C}$ and the mixture was stirred for further $3 \mathrm{~h}$ at the same temperature. The mixture was then quenched with aqueous saturated ammonium chloride and allowed to warm to $20^{\circ} \mathrm{C}$ over about $15 \mathrm{~min}$. Evaporation gave a pale yellow liquid that was partitioned between dichloromethane and aqueous $10 \%$ citric acid. The aqueous layer was extracted with dichloromethane $(3 \times 10 \mathrm{~mL})$ and all of the organic layers were combined, washed with saturated aqueous sodium hydrogen carbonate then brine, dried $\left(\mathrm{MgSO}_{4}\right)$, and evaporated. The residue was purified as described under the given product.

General procedure $\mathbf{E}$ for $\boldsymbol{N}$-deallylation. ${ }^{18 b, 19}$ Tris(triphenylphosphine)rhodium(I) chloride (Wilkinson's catalyst, $5 \mathrm{~mol} \%$ ) was added in one portion to a stirred solution of the $\beta$-amino ester (1.0 equiv.) in acetonitrile-water $(85: 15)$ at $20{ }^{\circ} \mathrm{C}$. The mixture was then heated under reflux for $16 \mathrm{~h}$. After allowing to cool, the mixture was extracted with dichloromethane, and the combined organic layers were dried $\left(\mathrm{MgSO}_{4}\right)$ and evaporated. The residue was purified as described under the given product.

Methyl 3-amino-2-methylpent-2-enoate (2d). Ammonium acetate $(2.60 \mathrm{~g}, 33.8 \mathrm{mmol})$ and acetic acid $(0.1 \mathrm{~mL})$ were added to a stirred solution of methyl 2-methyl-3-oxopentanoate (1d) $(0.98 \mathrm{~g}, 6.77 \mathrm{mmol})$ in benzene $(50 \mathrm{~mL})$. The mixture was heated under reflux for $72 \mathrm{~h}$ with the azeotropic removal of water. After allowing to cool to $20^{\circ} \mathrm{C}$, ethyl acetate $(50 \mathrm{~mL})$ was added and the mixture was washed with saturated aqueous sodium hydrogen carbonate $(15 \mathrm{~mL})$, dried $\left(\mathrm{Na}_{2} \mathrm{SO}_{4}\right)$, and evaporated to give $2 \mathbf{d}(0.51 \mathrm{~g}, 52 \%)$ as an orange oil; IR $v_{\max }$ 3303, 2924, 1744, 1656, 1610, 1462, $1161 \mathrm{~cm}^{-1}$; ${ }^{1} \mathrm{H} \mathrm{NMR}\left(300 \mathrm{MHz}, \mathrm{CDCl}_{3}\right) \delta 3.72(3 \mathrm{H}, \mathrm{s})$ $2.25(2 \mathrm{H}, \mathrm{q}, J=7.6 \mathrm{~Hz}) 1.75(3 \mathrm{H}, \mathrm{s}) 1.15(3 \mathrm{H}, \mathrm{t}, J=7.6 \mathrm{~Hz}) ; \mathrm{m} / z(\mathrm{CI}, \%) 144\left([\mathrm{M}+\mathrm{H}]^{+}, 65\right)$. HRMS $\mathrm{C}_{7} \mathrm{H}_{14} \mathrm{NO}_{2}$ calcd. 144.1025, found 144.1019.

Methyl 3-aminohexanoate (3c). Ammonium acetate (30.0 g, 0.39 mol) and acetic acid (0.1 $\mathrm{mL})$ were added to a stirred solution of methyl 3-oxohexanoate (1c) $(11.2 \mathrm{~g}, 77.7 \mathrm{mmol})$ in benzene $(160 \mathrm{~mL})$. The mixture was heated under reflux for $72 \mathrm{~h}$ with the azeotropic removal of water. After allowing to cool to $20^{\circ} \mathrm{C}$, the mixture was diluted with ethyl acetate (150 $\mathrm{mL})$, washed with saturated aqueous sodium hydrogen carbonate $(15 \mathrm{~mL})$, dried $\left(\mathrm{Na}_{2} \mathrm{SO}_{4}\right)$, 
and evaporated to give the crude $\beta$-enamino ester as an oil. Sodium borohydride (7.15 g, 0.19 mmol) was added in portions to a stirred solution of glacial acetic acid (200 mL) maintaining the temperature at near $20^{\circ} \mathrm{C}$. The mixture was stirred for $30 \mathrm{~min}$ until there was no more hydrogen was evolved. The $\beta$-enamino ester was then added in one portion and the mixture was stirred at $20^{\circ} \mathrm{C}$ for $3 \mathrm{~h}$. The acetic acid was removed under reduced pressure and the residue was dissolved in ethyl acetate $(100 \mathrm{~mL})$. The mixture was extracted with water $(4 \mathrm{x}$ $100 \mathrm{~mL}$ ), and the $\mathrm{pH}$ of the combined aqueous layers was adjusted to $\mathrm{pH} 12$ by potassium carbonate. The solution was extracted with chloroform $(3 \times 150 \mathrm{~mL})$ and the combined organic layers were dried $\left(\mathrm{MgSO}_{4}\right)$, and evaporated to give $\mathbf{3 c}(3.61 \mathrm{~g}, 32 \%)$ as an orange oil; IR $v_{\max } 3272,2957,1736,1553,1436,1379,1174 \mathrm{~cm}^{-1} ;{ }^{1} \mathrm{H}$ NMR $\left(300 \mathrm{MHz}, \mathrm{CDCl}_{3}\right) \delta 3.73$ $(3 \mathrm{H}, \mathrm{s}) 3.18(1 \mathrm{H}, \mathrm{m}) 2.46(1 \mathrm{H}, \mathrm{dd}, J=15.7,4.0 \mathrm{~Hz}) 2.25(1 \mathrm{H}, \mathrm{dd}, J=15.7,9.0 \mathrm{~Hz}) 2.03(2 \mathrm{H}$, br s) $1.36-1.33(4 \mathrm{H}, \mathrm{m}) 0.91(3 \mathrm{H}, \mathrm{t}, J=2.5 \mathrm{~Hz}) ;{ }^{13} \mathrm{C} \mathrm{NMR}\left(300 \mathrm{MHz}, \mathrm{CDCl}_{3}\right) \delta 173.1,51.5$, 48.0, 42.3, 39.7, 19.2, 14.0; m/z (CI, \%) $146\left([\mathrm{M}+\mathrm{H}]^{+}, 100\right)$. HRMS $\mathrm{C}_{7} \mathrm{H}_{16} \mathrm{NO}_{2}$ calcd. 146.1181, found 146.1183 .

Methyl 3-amino-2-methylpentanoate (3d). Sodium borohydride $(0.64 \mathrm{~g}, 16.8 \mathrm{mmol})$ was added in small portions to a stirred solution of glacial acetic acid $(11 \mathrm{~mL})$, maintaining the temperature near $20^{\circ} \mathrm{C}$. The mixture was then stirred for 30 min until no more hydrogen was evolved. Ester 2d (0.93 g, $6.47 \mathrm{mmol})$ was then added in one portion and the mixture was stirred at $20^{\circ} \mathrm{C}$ for $3 \mathrm{~h}$. The acetic acid was removed under reduced pressure and the residue was dissolved in ethyl acetate $(10 \mathrm{~mL})$. The solution was extracted with water $(4 \times 10 \mathrm{~mL})$, and the $\mathrm{pH}$ of the combined aqueous layers was adjusted to $\mathrm{pH} 12$ using potassium carbonate. The aqueous mixture was extracted with chloroform $(3 \times 15 \mathrm{~mL})$ and the combined organic layers were dried $\left(\mathrm{MgSO}_{4}\right)$, and evaporated to give $\mathbf{3 d}(0.63 \mathrm{~g}, 67 \%)$ as an orange oil, IR $v_{\max }$ $\left(\mathrm{cm}^{-1}\right)$ 3321, 2961, 1731, $1569 \mathrm{~cm}^{-1}$; ${ }^{1} \mathrm{H}$ NMR $\left(500 \mathrm{MHz}, \mathrm{CDCl}_{3}\right.$ ) (mixture of diastereoisomers) $\delta 3.67(3 \mathrm{H}, \mathrm{s}) 2.90-2.43(2 \mathrm{H}, \mathrm{m}) 1.53(2 \mathrm{H}, \mathrm{br} \mathrm{s}) 1.42-1.16(2 \mathrm{H}, \mathrm{m}) 1.14-$ $1.11(3 \mathrm{H}, \mathrm{m})$ 0.96-0.90 (3H, m). m/z (CI, \%) $146\left([\mathrm{M}+\mathrm{H}]^{+}, 100\right)$. HRMS $\mathrm{C}_{7} \mathrm{H}_{16} \mathrm{NO}_{2}$ calcd. 146.1181, found 146.1179 .

Methyl 3-amino-2-methyl-4-phenylbutanoate (3e). Trimethylsilyl chloride (0.26 mL, 2.0 mmol) was added to a stirred mixture of zinc dust $(2.60 \mathrm{~g}, 39.3 \mathrm{mmol})$ in dichloromethane (13 $\mathrm{mL})$ at $20^{\circ} \mathrm{C}$ under nitrogen, and the mixture was stirred for $30 \mathrm{~min}$. Tetrahydrofuran $(8 \mathrm{~mL})$ was then added and the mixture was heated to $42{ }^{\circ} \mathrm{C}$. A mixture of benzyl cyanide $(1.00 \mathrm{~g}$, $8.54 \mathrm{mmol})$ and methyl 2-bromopropanoate $(2.85 \mathrm{~g}, 17.1 \mathrm{mmol})$ was added and the reaction mixture was then heated under reflux for $2 \mathrm{~h}$. After allowing to cool the mixture was filtered, and sodium borohydride $(0.60 \mathrm{~g}, 15.4 \mathrm{mmol})$ and ethanol $(2.5 \mathrm{~mL})$ were added cautiously to the filtrate. The mixture was stirred for $3 \mathrm{~h}$, then hydrochloric acid $(2 \mathrm{M}, 9 \mathrm{~mL})$ was then 
added and the aqueous layer was extracted with dichloromethane $(2 \times 10 \mathrm{~mL})$. The combined organic layers were dried $\left(\mathrm{MgSO}_{4}\right)$ and evaporated. Toluene $(4 \mathrm{~mL})$ was added to the residue and the mixture was made alkaline with 0.880 aqueous ammonia $(3 \mathrm{~mL})$. The aqueous layer was extracted with toluene $(2 \times 4 \mathrm{~mL})$, and the organic layers were combined, dried $\left(\mathrm{MgSO}_{4}\right)$ and evaporated. The residue was purified by flash chromatography (20\% ethyl acetate/hexane) to give $3 \mathrm{e}(0.27 \mathrm{~g}, 15 \%)$ as a yellow oil; IR $v_{\max } 3368(\mathrm{~N}-\mathrm{H}), 2948(\mathrm{C}-\mathrm{H})$, 1726, $1664 \mathrm{~cm}^{-1} ;{ }^{1} \mathrm{H}$ NMR (500 MHz, $\left.\mathrm{CDCl}_{3}\right)$ (1:1 mixture of diastereoisomers) $\delta$ 7.38-7.30 $(5 \mathrm{H}, \mathrm{m}, \mathrm{Ph}) 3.69(3 \mathrm{H}, \mathrm{s}) 3.30(1 \mathrm{H}, \mathrm{m}) 2.82(1 \mathrm{H}, \mathrm{m}) 2.60-2.40(2 \mathrm{H}, \mathrm{m}) 1.65(2 \mathrm{H}, \mathrm{br} \mathrm{s}) 1.26-$ 1.18 (3H, m); ${ }^{13} \mathrm{C}$ NMR (500 MHz, $\mathrm{CDCl}_{3}$ ) $\delta$ 176.1, 175.9, 139.1, 139.1, 129.4, 129.3, 129.1, 128.6, 126.5, 55.5, 54.4, 51.7, 51.6, 45.7, 44.6, 41.8, 41.6, 14.5, 11.7; m/z (CI, \%) 208 $\left([\mathrm{M}+\mathrm{H}]^{+}, 100\right)$. HRMS $\mathrm{C}_{12} \mathrm{H}_{18} \mathrm{NO}_{2}$ calcd. 208.1338, found 208.1335.

Dihydrochloride salt of methyl 3-amino-3-(pyridin-3-yl)propanoate (3g). 3-Amino-3(pyridin-3-yl)propanoic acid was prepared as previously described ${ }^{31}$ and directly treated with thionyl chloride in methanol to give the dihydrochlorde salt of $\mathbf{3 g}$. To a stirred solution of pyridine-3-carboxaldehyde $(8.24 \mathrm{~g}, 76.8 \mathrm{mmol})$ in ethanol $(15 \mathrm{~mL})$ were added malonic acid $(8.0 \mathrm{~g}, 76.8 \mathrm{mmol})$ and ammonium acetate $(12.0 \mathrm{~g}, 0.156 \mathrm{mmol})$. The mixture was heated under reflux for $6 \mathrm{~h}$. After allowing to cool, the mixture was filtered and the filtrate evaporated. The residue was dissolved in methanol $(150 \mathrm{~mL})$ and the solution cooled to $0{ }^{\circ} \mathrm{C}$. To the stirred solution thionyl chloride $(2.7 \mathrm{~mL}, 99.8 \mathrm{mmol})$ was added dropwise; the mixture was stirred at $0{ }^{\circ} \mathrm{C}$ for an additional $30 \mathrm{~min}$, then stirred at $20{ }^{\circ} \mathrm{C}$ for $3 \mathrm{~h}$. The mixture was then heated at reflux for $1 \mathrm{~h}$. After allowing to cool, diethyl ether $(200 \mathrm{~mL})$ was added and the precipitate filtered to give the dihydrochloride salt of $\mathbf{3 g}(5.42 \mathrm{~g}, 43 \%)$ as a white solid, $\mathrm{mp}$ 202-205 ${ }^{\circ} \mathrm{C}$ (lit. ${ }^{32} \mathrm{mp} 197.5-199{ }^{\circ} \mathrm{C}$ ); IR $v_{\max } 3296,2953,1737,1657 \mathrm{~cm}^{-1} ;{ }^{1} \mathrm{H}$ NMR (500 $\left.\mathrm{MHz}, \mathrm{D}_{2} \mathrm{O}\right) \delta 8.54(1 \mathrm{H}, \mathrm{m}) 8.50(1 \mathrm{H}, \mathrm{m}) 7.90(1 \mathrm{H}, \mathrm{m}) 7.47(1 \mathrm{H}, \mathrm{m}) 4.74(1 \mathrm{H}, \mathrm{dd}, J=7.9,6.6$ Hz) $3.70(3 \mathrm{H}, \mathrm{s}) 2.88(1 \mathrm{H}, \mathrm{dd}, J=16.3,7.9 \mathrm{~Hz}) 2.80(1 \mathrm{H}, \mathrm{dd}, J=16.3,6.6 \mathrm{~Hz})$.

Methyl 3-(2-methoxycarbonylacetylamino)butanoate (4a). Following general procedure A, reaction of methyl 3-aminobutanoate hydrochloride $(0.50 \mathrm{~g}, 3.3 \mathrm{mmol})$ in dichloromethane $(7$ $\mathrm{mL})$, 1-hydroxybenzotriazole (0.66 g, $4.9 \mathrm{mmol}), N, N$-diisopropylethylamine (2.3 $\mathrm{mL}, 13$ mmol), $N$-(3-dimethylaminopropyl)- $N$ '-ethylcarbodiimide hydrochloride $(0.59 \mathrm{~g}, 3.3 \mathrm{mmol})$, and monomethyl malonate $(1.52 \mathrm{~g}, 9.8 \mathrm{mmol})$ in dichloromethane $(10 \mathrm{~mL})$ gave a pale yellow solid that was purified by flash chromatography (60\% ethyl acetate/hexane) to give $\mathbf{4 a}(0.33$ $\mathrm{g}, 46 \%)$ as a yellow gum; IR $v_{\max } 3293,2956,1730,1650 \mathrm{~cm}^{-1} ;{ }^{1} \mathrm{H} \mathrm{NMR}\left(500 \mathrm{MHz}, \mathrm{CDCl}_{3}\right) \delta$ $7.26(1 \mathrm{H}, \mathrm{br} \mathrm{s}) 4.35(1 \mathrm{H}, \mathrm{m}) 3.63(3 \mathrm{H}, \mathrm{s}) 3.58(3 \mathrm{H}, \mathrm{s}) 3.19(2 \mathrm{H}, \mathrm{s}) 2.51-2.36(2 \mathrm{H}, \mathrm{m}) 1.13$ $(3 \mathrm{H}, \mathrm{d}, J=6.7 \mathrm{~Hz}) ;{ }^{13} \mathrm{C}$ NMR $\left(500 \mathrm{MHz}, \mathrm{CDCl}_{3}\right) \delta 171.7,169.4,164.3,52.3,51.6,42.2,41.3$, 
39.8, 19.8; m/z (CI, \%) $218\left([\mathrm{M}+\mathrm{H}]^{+}, 100\right)$. HRMS $\mathrm{C}_{9} \mathrm{H}_{16} \mathrm{NO}_{5}$ calcd. 218.1023, found 218.1022.

Methyl 3-(2-methoxycarbonylacetylamino)-hexanoate (4c). Following general procedure A, reaction of methyl 3-aminohexanoate $3 \mathrm{c}(0.47 \mathrm{~g}, 3.3 \mathrm{mmol})$ in dichloromethane $(7 \mathrm{~mL}), 1$ hydroxybenzotriazole $(0.66 \mathrm{~g}, 4.9 \mathrm{mmol}), N, N$-diisopropylethylamine $(2.3 \mathrm{~mL}, 13 \mathrm{mmol}), N$ (3-dimethylaminopropyl)- $N$ '-ethylcarbodiimide hydrochloride $(0.59 \mathrm{~g}, 3.3 \mathrm{mmol})$, and monomethyl malonate $(1.52 \mathrm{~g}, 9.8 \mathrm{mmol})$ in dichloromethane $(10 \mathrm{~mL})$ gave a pale yellow solid that was purified by flash chromatography (55:45 ethyl acetate/hexane) to give $\mathbf{4 c}(0.32$ $\mathrm{g}, 40 \%$ ) as a colourless gum; IR $v_{\max } 3292,2956,1734,1649 \mathrm{~cm}^{-1} ;{ }^{1} \mathrm{H}$ NMR (300 MHz, $\left.\mathrm{CDCl}_{3}\right) \delta 7.22(1 \mathrm{H}, \mathrm{br} \mathrm{s}) 4.38(1 \mathrm{H}, \mathrm{m}) 3.74(3 \mathrm{H}, \mathrm{s}) 3.69(3 \mathrm{H}, \mathrm{s}) 3.31(2 \mathrm{H}, \mathrm{s}) 2.56-2.54(2 \mathrm{H}$, m) $1.56-1.49(2 \mathrm{H}, \mathrm{m}) 1.35(2 \mathrm{H}, \mathrm{m}) 0.91(3 \mathrm{H}, \mathrm{t}, J=7.3 \mathrm{~Hz}) ;{ }^{13} \mathrm{C} \mathrm{NMR}\left(300 \mathrm{MHz}, \mathrm{CDCl}_{3}\right) \delta$ 172.1, 169.7, 164.3, 52.4, 51.7, 46.0, 41.2, 38.4, 36.1, 19.3, 13.8; $\mathrm{m} / \mathrm{z}(\mathrm{CI}, \%) 246\left([\mathrm{M}+\mathrm{H}]^{+}\right.$, 100). HRMS $\mathrm{C}_{11} \mathrm{H}_{20} \mathrm{NO}_{5}$ calcd. 246.1336, found 246.1332.

Methyl 3-(2-methoxycarbonylacetylamino)-2-methylpentanoate (4d). Following general procedure B, reaction of ester $\mathbf{3 d}(0.30 \mathrm{~g}, 2.07 \mathrm{mmol})$, triethylamine $(0.38 \mathrm{~mL}, 2.7 \mathrm{mmol})$, and methyl 3-chloro-3-oxopropanoate $(0.27 \mathrm{~mL}, 2.48 \mathrm{mmol})$ in dichloromethane $(8 \mathrm{~mL})$ gave a pale yellow oil was purified by flash chromatography (40:60 ethyl acetate/hexane) to give 4d $(0.20 \mathrm{~g}, 40 \%)$ as a pale yellow oil; ${ }^{1} \mathrm{H}$ NMR (300 $\mathrm{MHz}, \mathrm{CDCl}_{3}$ ) (mixture of diastereoisomers) $\delta 7.35(1 \mathrm{H}, \mathrm{m}) 4.17(1 \mathrm{H}, \mathrm{m}) 3.75(3 \mathrm{H}, \mathrm{s}) 3.67(3 \mathrm{H}, \mathrm{s}) 3.34-3.30(2 \mathrm{H}, \mathrm{m})$ $2.68(1 \mathrm{H}, \mathrm{m}) 1.61-1.55(2 \mathrm{H}, \mathrm{m}) 1.16(3 \mathrm{H}, \mathrm{d}, J=6.0 \mathrm{~Hz}) 0.92(3 \mathrm{H}, \mathrm{t}, J=7.2 \mathrm{~Hz}) ;{ }^{13} \mathrm{C}$ NMR $\left(300 \mathrm{MHz}, \mathrm{CDCl}_{3}\right) \delta 175.7,174.8,169.6,169.3,165.0,164.8,52.8,52.7,52.4,52.3,51.7$, 48.6, 43.3, 42.0, 41.5, 41.2, 26.4, 24.7, 14.7, 12.8, 10.6; $\mathrm{m} / \mathrm{z}(\mathrm{CI}, \%) 246\left([\mathrm{M}+\mathrm{H}]^{+}, 100\right)$. HRMS $\mathrm{C}_{11} \mathrm{H}_{20} \mathrm{NO}_{5}$ calcd. 246.1342, found 246.1345.

Methyl 3-(2-methoxycarbonylacetylamino)-2-methyl-4-phenylbutanoate (4e). Following general procedure $\mathrm{B}$, reaction of amine $3 \mathbf{e}(0.13 \mathrm{~g}, 0.60 \mathrm{mmol}), N, N$-diisopropylethylamine $(0.21 \mathrm{~mL}, 1.21 \mathrm{mmol})$, and methyl 3-chloro-3-oxopropanoate $(0.10 \mathrm{~mL}, 0.90 \mathrm{mmol})$ in dichloromethane $(4 \mathrm{~mL})$ afforded an oil that was purified by flash chromatography (40:60 ethyl acetate:hexane) to give $4 \mathbf{e}(0.13 \mathrm{~g}, 67 \%)$ as a colourless oil; IR $v_{\max } 3301,2953,1733$, $1657 \mathrm{~cm}^{-1} ;{ }^{1} \mathrm{H}$ NMR (500 MHz, $\mathrm{CDCl}_{3}$ ) (mixture of diastereoisomers) $\delta$ 7.29-7.16 (5H, m) 4.51-4.13 $(1 \mathrm{H}, \mathrm{m}) 3.72(3 \mathrm{H}, \mathrm{s}) 3.66(3 \mathrm{H}, \mathrm{s}) 3.27-3.12(2 \mathrm{H}, \mathrm{m}) 2.84-2.67(3 \mathrm{H}, \mathrm{m}) 1.22-1.18$ $(3 \mathrm{H}, \mathrm{m}) ;{ }^{13} \mathrm{C} \mathrm{NMR}\left(500 \mathrm{MHz}, \mathrm{CDCl}_{3}\right) \delta 175.9,174.8,169.8,169.4,164.8,164.3,137.8$, 137.6, 129.3, 129.2, 128.5, 128.4, 126.7, 126.6, 53.1, 52.4, 52.3, 51.9, 42.9, 42.6, 41.5, 41.0, 40.7, 39.9, 37.8, 37.7, 13.2, 12.9; $\mathrm{m} / \mathrm{z}(\mathrm{EI}, \%) 308\left([\mathrm{M}+\mathrm{H}]^{+}, 5\right), 276\left(\left[\mathrm{M}-\mathrm{CH}_{4} \mathrm{O}\right]^{+}, 12\right) . \mathrm{HRMS}$ $\mathrm{C}_{16} \mathrm{H}_{22} \mathrm{NO}_{5}$ calcd. 308.1498 , found 308.1491 . 
Methyl 3-(2-methoxycarbonylacetylamino)-4-(pyridin-3-yl)butanoate (4g). Following general procedure B, reaction of amine $3 \mathrm{~g}(0.50 \mathrm{~g}, 1.98 \mathrm{mmol}), N, N$-diisopropylethylamine (1.4 mL, $7.93 \mathrm{mmol})$, and methyl 3-chloro-3-oxopropanoate $(0.32 \mathrm{~mL}, 2.98 \mathrm{mmol})$ in dichloromethane $(12 \mathrm{~mL})$ gave an oil was purified by flash chromatography (1:1 ethyl acetate:hexane) to give $\mathbf{4 g}(0.54 \mathrm{~g}, 97 \%)$ as a yellow oil; IR $v_{\max } 3273,2954,1733,1656 \mathrm{~cm}^{-1}$; ${ }^{1} \mathrm{H}$ NMR $\left(500 \mathrm{MHz}, \mathrm{CDCl}_{3}\right)$ (mixture of rotamers) $\delta 8.67(1 \mathrm{H}, \mathrm{s}) 8.53(1 \mathrm{H}, \mathrm{m}) 7.64-7.55(1 \mathrm{H}$, m) $7.22(1 \mathrm{H}, \mathrm{m}) 5.38(1 \mathrm{H}, \mathrm{dd}, J=6.4,5.3 \mathrm{~Hz}) 3.67(3 \mathrm{H}, \mathrm{s}) 3.53(3 \mathrm{H}, \mathrm{s}) 3.26(2 \mathrm{H}, \mathrm{s}) 2.86(1 \mathrm{H}$, $\mathrm{dd}, J=16.0,6.4 \mathrm{~Hz}) 2.79(1 \mathrm{H}, \mathrm{dd}, J=16.0,5.3 \mathrm{~Hz}) ;{ }^{13} \mathrm{C} \mathrm{NMR}\left(500 \mathrm{MHz}, \mathrm{CDCl}_{3}\right) \delta 170.8$, 169.3, 164.8, 148.8, 148.1, 136.2, 134.4, 123.6, 123.3, 52.5, 52.0, 47.9, 41.3, 39.6; $\mathrm{m} / \mathrm{z}$ (EI, \%) $281\left([\mathrm{M}+\mathrm{H}]^{+}, 51\right), 280\left(\mathrm{M}^{+}, 88\right)$. HRMS $\mathrm{C}_{13} \mathrm{H}_{17} \mathrm{~N}_{2} \mathrm{O}_{5}$ calcd. 281.1137, found 281.1136.

3-Methoxycarbonyl-6-phenylpiperidine-2,4-dione (5f). Sodium methoxide in methanol (2.0 M, $3.1 \mathrm{~mL}, 6.2 \mathrm{mmol})$ was added to a stirred solution of diester $\mathbf{4} \mathbf{f}^{25}(1.40 \mathrm{~g}, 5.0 \mathrm{mmol})$ in methanol $(15 \mathrm{~mL})$ at $20^{\circ} \mathrm{C}$ under nitrogen. The mixture was then heated under reflux for $2 \mathrm{~h}$. After allowing to cool to $20{ }^{\circ} \mathrm{C}$, the mixture was diluted with diethyl ether and filtered. The white precipitate was dissolved in water, and the solution acidified to $\mathrm{pH}$ 2-3 by hydrochloric acid $(1 \mathrm{M})$. After extraction with ethyl acetate $(3 \times 20 \mathrm{~mL})$ the combined organic layers were washed with brine $(10 \mathrm{~mL})$, dried $\left(\mathrm{MgSO}_{4}\right)$ and evaporated to give $\mathbf{5 f}(0.86 \mathrm{~g}, 70 \%)$ as a white solid, mp 122-125 ${ }^{\circ} \mathrm{C}$ (lit. ${ }^{13} 128-130{ }^{\circ} \mathrm{C}$ ); IR $v_{\max } 3311(\mathrm{O}-\mathrm{H}), 2952,1713,1638,1571 \mathrm{~cm}^{-1}$; ${ }^{1} \mathrm{H}$ NMR $\left(500 \mathrm{MHz}, \mathrm{CDCl}_{3}\right)$ ठ 7.27-7.14 (5H, m) $6.22(1 \mathrm{H}, \mathrm{br} \mathrm{s}) 4.64(1 \mathrm{H}, \mathrm{t}, J=7.5 \mathrm{~Hz}) 3.68$ $(3 \mathrm{H}, \mathrm{s}) 2.81(2 \mathrm{H}, \mathrm{d}, J=7.5 \mathrm{~Hz}) ;{ }^{13} \mathrm{C} \mathrm{NMR}\left(500 \mathrm{MHz}, \mathrm{CDCl}_{3}\right) \delta 183.5,172.0,164.8,139.6$, $129.1,128.7,126.5,97.4,52.7,52.3,37.8$.

6-Methylpiperidine-2,4-dione (6a). Following general procedure $\mathrm{C}$, reaction of diester 4a $(0.30 \mathrm{~g}, 1.38 \mathrm{mmol})$, and sodium methoxide in methanol $(1.84 \mathrm{M}, 1.5 \mathrm{~mL}, 2.76 \mathrm{mmol})$ in methanol $(2.0 \mathrm{~mL})$ gave $6 \mathbf{a}(74 \mathrm{mg}, 42 \%)$ as a pale yellow solid, $\mathrm{mp} 124-128{ }^{\circ} \mathrm{C}$; IR $v_{\max }$ 3217, 2968, 1726, 1663, cm ${ }^{-1}$; ${ }^{1} \mathrm{H}$ NMR $\left(500 \mathrm{MHz}, \mathrm{CDCl}_{3}\right) \delta 3.88(1 \mathrm{H}, \mathrm{m}) 3.26(2 \mathrm{H}, \mathrm{d}, J=$ $19.8 \mathrm{~Hz}) 3.19(1 \mathrm{H}, \mathrm{d}, J=19.8 \mathrm{~Hz}) 2.64(1 \mathrm{H}, \mathrm{dd}, J=16.4,4.0 \mathrm{~Hz}) 2.30(1 \mathrm{H}, \mathrm{dd}, J=16.4,9.6$ Hz) $1.30(3 \mathrm{H}, \mathrm{d}, J=6.5 \mathrm{~Hz}) ;{ }^{13} \mathrm{C}$ NMR $\left(500 \mathrm{MHz}, \mathrm{CDCl}_{3}\right) \delta 203.4,169.8,47.0,46.2,44.6$, $21.3 ; \mathrm{m} / \mathrm{z}(\mathrm{CI}, \%) 128\left([\mathrm{M}+\mathrm{H}]^{+}, 100\right)$. HRMS $\mathrm{C}_{6} \mathrm{H}_{10} \mathrm{NO}_{2}$ calcd. 128.0711, found 128.0714.

6-Propylpiperidine-2,4-dione (6c). Following general procedure $C$, reaction of diester 4c $(0.17 \mathrm{~g}, 0.70 \mathrm{mmol})$, and sodium methoxide in methanol $(2.0 \mathrm{M}, 0.54 \mathrm{~mL}, 1.05 \mathrm{mmol})$ in methanol $(1.0 \mathrm{~mL})$ gave $6 \mathrm{c}(63 \mathrm{mg}, 58 \%)$ as a white solid, $\mathrm{mp} 112-116{ }^{\circ} \mathrm{C}$; IR $v_{\max } 3249$, 2925, 1736, $1664 \mathrm{~cm}^{-1}$; ${ }^{1} \mathrm{H}$ NMR (300 MHz, $\left.\mathrm{CDCl}_{3}\right) \delta 3.65(1 \mathrm{H}, \mathrm{m}) 3.25(2 \mathrm{H}, \mathrm{m}) 2.66(1 \mathrm{H}$,

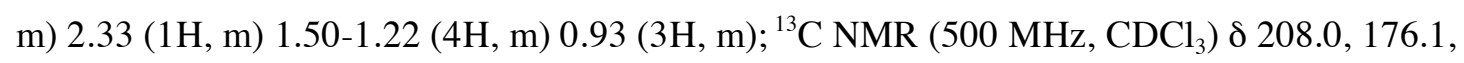


36.9, 34.0, 29.7, 21.1, 18.4, 13.9; $\mathrm{m} / z(\mathrm{CI}, \%) 156\left([\mathrm{M}+\mathrm{H}]^{+}\right.$, 35). HRMS $\mathrm{C}_{8} \mathrm{H}_{14} \mathrm{NO}_{2}$ calcd. 156.1025, found 156.1022 .

6-Ethyl-5-methylpiperidine-2,4-dione (6d). Following general procedure $\mathrm{C}$, reaction of diester $5 \mathbf{d}(0.12 \mathrm{~g}, 0.48 \mathrm{mmol})$, and sodium methoxide in methanol $(1.74 \mathrm{M}, 0.53 \mathrm{~mL}, 0.92$ mmol) in methanol (3.0 mL) gave $\mathbf{6 d}(53 \mathrm{mg}, 71 \%)$ as a yellow oil; IR $v_{\max } 3280,2925,1725$, $1661 \mathrm{~cm}^{-1} ;{ }^{1} \mathrm{H}$ NMR $\left(300 \mathrm{MHz}, \mathrm{CDCl}_{3}\right.$ ) (mixture of diastereoisomers) $\delta$ 7.95-7.79 (1H, br s) 3.75-3.69 $(1 \mathrm{H}, \mathrm{m}) 3.22(2 \mathrm{H}, \mathrm{m})$ 2.67-2.36 $(1 \mathrm{H}, \mathrm{m}) 1.73-1.52(2 \mathrm{H}, \mathrm{m})$ 1.19-1.05 (3H, m) 0.99$0.83(3 \mathrm{H}, \mathrm{m}) ;{ }^{13} \mathrm{C} \mathrm{NMR}\left(300 \mathrm{MHz}, \mathrm{CDCl}_{3}\right) \delta 205.7,170.0,55.6,54.5,46.4,46.4,46.0,41.0$, 26.2, 24.4, 11.6, 10.1, 10.0, 8.5; m/z (CI, \%) $156\left([\mathrm{M}+\mathrm{H}]^{+}, 100\right)$. HRMS $\mathrm{C}_{8} \mathrm{H}_{14} \mathrm{NO}_{2}$ calcd. 156.2023 , found 156.2021 .

6-Benzyl-5-methylpiperidine-2,4-dione (6e). Following general procedure $\mathrm{C}$, reaction of diester 5e $(0.09 \mathrm{~g}, 0.30 \mathrm{mmol})$, and sodium methoxide in methanol $(2.0 \mathrm{M}, 0.2 \mathrm{~mL}, 0.38$ $\mathrm{mmol}$ ) in methanol $(1.2 \mathrm{~mL})$ gave $6 \mathrm{e}(36 \mathrm{mg}, 56 \%)$ as a yellow oil, IR $v_{\max } 3245(\mathrm{~N}-\mathrm{H}), 2923$, 1721, $1661 \mathrm{~cm}^{-1} ;{ }^{1} \mathrm{H}$ NMR (500 MHz, $\mathrm{CDCl}_{3}$ ) (mixture of diastereoisomers) $\delta 7.63-7.19(5 \mathrm{H}$, m) 3.92-3.83 $(1 \mathrm{H}, \mathrm{m}) 3.27-3.13(2 \mathrm{H}, \mathrm{m}) 2.90(1 \mathrm{H}, \mathrm{m}) 2.65-2.61(1 \mathrm{H}, \mathrm{m}) 2.51-2.46(1 \mathrm{H}, \mathrm{m})$ 1.28-1.25 (3H, m); ${ }^{13} \mathrm{C}$ NMR (500 MHz, $\mathrm{CDCl}_{3}$ ) $\delta$ 204.8, 204.7, 169.2, 168.4, 136.0, 135.5, 129.5, 129.4, 129.3, 129.1, 127.7, 127.4, 55.8, 54.5, 47.4, 46.6, 46.1, 46.0, 41.0, 37.9, 12.1, $10.5 ; \mathrm{m} / z(\mathrm{CI}, \%) 218\left(\mathrm{M}+\mathrm{H}^{+}, 100\right)$. HRMS $\mathrm{C}_{13} \mathrm{H}_{16} \mathrm{NO}_{2}$ calcd. 218.1181, found 218.1189.

6-Phenylpiperidine-2,4-dione (6f). Diester $\mathbf{5 f}(0.31 \mathrm{~g}, 1.25 \mathrm{mmol})$ was added to $1 \%$ water in acetonitrile $(6 \mathrm{~mL})$. The mixture was heated under reflux for $2 \mathrm{~h}$, allowed to cool and then evaporated. The residue was purified by flash chromatography (1:99 methanol:chloroform) to give $6 \mathbf{f}(0.25 \mathrm{~g}, 95 \%)$ as a white solid, mp $160-163{ }^{\circ} \mathrm{C}\left(\right.$ lit. $\left.^{33} 167-169{ }^{\circ} \mathrm{C}\right)$; IR $v_{\max } 3248,2927$, $1722,1664 \mathrm{~cm}^{-1} ;{ }^{1} \mathrm{H}$ NMR $\left(500 \mathrm{MHz}, \mathrm{CDCl}_{3}\right) \delta$ 7.47-7.28 $(5 \mathrm{H}, \mathrm{m}) 6.87(1 \mathrm{H}$, br s) $4.80(1 \mathrm{H}$, $\mathrm{dd}, J=8.7,4.5 \mathrm{~Hz}) 3.34(2 \mathrm{H}, \mathrm{s}) 2.88(1 \mathrm{H}, \mathrm{dd}, J=16.0,4.5 \mathrm{~Hz}) 2.88(1 \mathrm{H}, \mathrm{dd}, J=16.0,8.7 \mathrm{~Hz})$; ${ }^{13} \mathrm{C}$ NMR $\left(500 \mathrm{MHz}, \mathrm{CDCl}_{3}\right.$ ) $\delta 202.3,169.0,139.3,129.4,128.9,126.0,52.9,47.2,47.0 ; \mathrm{m} / \mathrm{z}$ $(\mathrm{CI}, \%) 190\left([\mathrm{M}+\mathrm{H}]^{+}, 95\right)$. HRMS $\mathrm{C}_{11} \mathrm{H}_{12} \mathrm{NO}_{2}$ calcd. 190.0863, found 190.0859.

3,3-Dimethyl-6-phenylpiperidine-2,4-dione (6h). Potassium carbonate $(0.25 \mathrm{~g}, 1.84 \mathrm{mmol})$ and methyl iodide $(0.11 \mathrm{~mL}, 1.84 \mathrm{mmol})$ were added to a solution of 6-phenylpiperidine-2,4dione $(\mathbf{6 f})(0.12 \mathrm{~g}, 0.61 \mathrm{mmol})$ in acetone $(3 \mathrm{~mL})$. The mixture was stirred at $50{ }^{\circ} \mathrm{C}$ for $16 \mathrm{~h}$. Filtration and evaporation of the filtrate gave a residue that was purified by flash chromatography (60:40 ethyl acetate/hexane) to give $\mathbf{6 h}(0.09 \mathrm{~g}, 65 \%)$ as a white solid, $\mathrm{mp}$ 165-168 ${ }^{\circ} \mathrm{C}$ (lit. ${ }^{34} 168-169{ }^{\circ} \mathrm{C}$ ); IR $v_{\max } 3200,2978,1714,1649 \mathrm{~cm}^{-1}$; ${ }^{1} \mathrm{H}$ NMR $(300 \mathrm{MHz}$, $\left.\mathrm{CDCl}_{3}\right) \delta 7.60-7.26(5 \mathrm{H}, \mathrm{m}) 6.60(1 \mathrm{H}, \mathrm{br} \mathrm{s}) 4.70(1 \mathrm{H}, \mathrm{dd}, J=4.7,2.5 \mathrm{~Hz}) 2.92(1 \mathrm{H}, \mathrm{dd}, J=$ 
15.5, 4.7 Hz) $2.84(1 \mathrm{H}, \mathrm{dd}, J=15.5,2.5 \mathrm{~Hz}) 1.39(3 \mathrm{H}, \mathrm{s}) 1.38(3 \mathrm{H}, \mathrm{s}) ;{ }^{13} \mathrm{C} \mathrm{NMR}(300 \mathrm{MHz}$, $\left.\mathrm{CDCl}_{3}\right) \delta 208.2,175.8,140.0,129.3,128.8,126.0,52.1,52.0,45.3,23.1,22.8$.

3-Methoxycarbonyl-3-methyl-6-phenylpiperidine-2,4-dione (6i). Tetrabutylammonium fluoride in THF $(1 \mathrm{M}, 0.8 \mathrm{~mL})$ and methyl iodide $(0.08 \mathrm{~mL}, 1.28 \mathrm{mmol})$ were added to 3 methoxycarbonyl-6-phenylpiperidine-2,4-dione (5f) $(0.16 \mathrm{~g}, 0.64 \mathrm{mmol})$ in THF $(2 \mathrm{~mL})$. The mixture was stirred at $20{ }^{\circ} \mathrm{C}$ for $24 \mathrm{~h}$. The solution was neutralised with hydrochloric acid (1M), extracted with chloroform and evaporated. The residue that was purified by flash chromatography (55:45 ethyl acetate:hexane) to give $\mathbf{6 i}(0.11 \mathrm{~g}, 64 \%)$ as a colourless solid; ${ }^{1} \mathrm{H}$ NMR (300 MHz, $\left.\mathrm{CDCl}_{3}\right)(1: 1$ mixture of diastereoisomers) $\delta 7.47-7.21(5 \mathrm{H}, \mathrm{m}) 6.60(1 \mathrm{H}, \mathrm{br}$ s) $4.82-4.49(1 \mathrm{H}, \mathrm{m}) 3.69$ and $3.64(3 \mathrm{H}, \mathrm{s}) 3.04-2.73(2 \mathrm{H}, \mathrm{m}) 1.57$ and $1.51(3 \mathrm{H}, \mathrm{s}) ;{ }^{13} \mathrm{C} \mathrm{NMR}$ (300 MHz, $\mathrm{CDCl}_{3}$ ) $\delta$ 201.7, 201.6, 170.3, 169.6, 167.6, 167.5, 139.3, 139.2, 129.4, 129.3, 129.0, 128.9, 126.2, 126.1, 63.8, 63.7, 53.5, 53.4, 52.4, 52.0, 45.7, 45.6, 18.3, 18.2; $\mathrm{m} / \mathrm{z}$ (CI, \%) $262\left([\mathrm{M}+\mathrm{H}]^{+}, 100\right)$. HRMS C ${ }_{14} \mathrm{H}_{16} \mathrm{NO}_{4}$ calcd. 262.1074, found 262.1067.

Methyl (3S, $\alpha S)-3-[N$-allyl- $N$-( $\alpha$-methylbenzyl)]aminobutanoate (8a). Following general procedure $\mathrm{D}$, butyllithium in hexanes $(1.6 \mathrm{M}, 8.0 \mathrm{~mL}, 12.9 \mathrm{mmol})$ and methyl but-2-enoate $(0.83 \mathrm{~g}, \quad 8.3 \mathrm{mmol})$ in anhydrous THF $(9 \mathrm{~mL})$ were added to $(S)-N-(\alpha-$ methylbenzyl)allylamine (7) $(2.15 \mathrm{~g}, 13.3 \mathrm{mmol})$ in anhydrous THF $(18 \mathrm{~mL})$, affording an oil that was purified by flash chromatography (15:85 ethyl acetate:hexane) to give 8a (1.85 g, $85 \%)$ as a pale yellow oil; $[\alpha]_{\mathrm{D}}{ }^{21}=+18.7\left(c 0.75, \mathrm{CHCl}_{3}\right)$, lit. $^{35}$ for enantiomer $[\alpha]_{\mathrm{D}}{ }^{26}=-14.2$ (c 1.85, $\mathrm{CHCl}_{3}$ ); IR $v_{\max } 2970,1737 \mathrm{~cm}^{-1} ;{ }^{1} \mathrm{H} \mathrm{NMR}\left(500 \mathrm{MHz}, \mathrm{CDCl}_{3}\right) \delta$ 7.37-7.20 (5H, m) $5.85(1 \mathrm{H}, \mathrm{m}) 5.15(1 \mathrm{H}, \mathrm{dd}, J=17.2,10.4 \mathrm{~Hz}) 5.04(1 \mathrm{H}, \mathrm{dd}, J=17.2,4.6 \mathrm{~Hz}) 3.97(1 \mathrm{H}, \mathrm{q}, J=$ $6.8 \mathrm{~Hz}) 3.56(3 \mathrm{H}, \mathrm{s}) 3.49(1 \mathrm{H}, \mathrm{m}) 3.18(2 \mathrm{H}, \mathrm{d}, J=6.3 \mathrm{~Hz}) 2.40(1 \mathrm{H}, \mathrm{dd}, J=14.2,7.0 \mathrm{~Hz}) 2.19$ $(1 \mathrm{H}, \mathrm{dd}, J=14.2,7.5 \mathrm{~Hz}) 1.37(3 \mathrm{H}, \mathrm{d}, J=6.8 \mathrm{~Hz}) 1.07(3 \mathrm{H}, \mathrm{d}, J=6.8 \mathrm{~Hz}) ;{ }^{13} \mathrm{C}$ NMR $(500$ $\left.\mathrm{MHz}, \mathrm{CDCl}_{3}\right) \delta 172.9,145.2,139.2,128.1,127.6,126.8,115.6,57.7,51.4,50.6,48.6,40.4$, 18.8, 17.5; m/z (CI, \%) $262\left([\mathrm{M}+\mathrm{H}]^{+}\right)$. HRMS C ${ }_{16} \mathrm{H}_{24} \mathrm{NO}_{2}$ calcd. 262.1807, found 262.1803.

\section{Methyl $\quad(3 R, \alpha S)-3-[N$-allyl- $N$-( $\alpha$-methylbenzyl)amino]-4-methylpentanoate}

(8b).

Following general procedure $\mathrm{D}$, butyllithium in hexanes $(2.5 \mathrm{M}, 5.3 \mathrm{~mL}, 13.3 \mathrm{mmol})$ and methyl 4-methylpent-2-enoate $(1.10 \mathrm{~g}, 8.58 \mathrm{mmol})$ in anhydrous THF $(22 \mathrm{~mL})$ were added to (S)- $N$-( $\alpha$-methylbenzyl)allylamine (7) $(2.22 \mathrm{~g}, 13.7 \mathrm{mmol})$ in anhydrous THF $(28 \mathrm{~mL})$, affording an oil that was purified by flash chromatography (12:88 ethyl acetate:hexane) to give $\mathbf{8 b}(1.70 \mathrm{~g}, 69 \%)$ as a pale yellow oil; $[\alpha]_{\mathrm{D}}^{21}=+18.0\left(c 5.00, \mathrm{CHCl}_{3}\right)$; IR $v_{\max } 2970,1736$ $\mathrm{cm}^{-1} ;{ }^{1} \mathrm{H}$ NMR $\left(500 \mathrm{MHz}, \mathrm{CDCl}_{3}\right)$ d 7.31-7.21 $(5 \mathrm{H}, \mathrm{m}) 5.90(1 \mathrm{H}, \mathrm{m}) 5.20(1 \mathrm{H}, \mathrm{m}) 5.07(1 \mathrm{H}$, m) $3.91(1 \mathrm{H}, \mathrm{q}, J=7.1 \mathrm{~Hz}) 3.58(3 \mathrm{H}, \mathrm{s}) 3.18(1 \mathrm{H}, \mathrm{m}) 3.09-3.06(2 \mathrm{H}, \mathrm{m}) 2.11(1 \mathrm{H}, \mathrm{dd}, J=15.6$, $7.8 \mathrm{~Hz}) 2.02(1 \mathrm{H}, \mathrm{dd}, J=15.6,4.0 \mathrm{~Hz}) 1.69(1 \mathrm{H}, \mathrm{m}) 1.43(3 \mathrm{H}, \mathrm{d}, J=7.1 \mathrm{~Hz}) 0.98(3 \mathrm{H}, \mathrm{d}, J=$ 
$6.7 \mathrm{~Hz}) 0.81(3 \mathrm{H}, \mathrm{d}, J=6.7 \mathrm{~Hz}) ;{ }^{13} \mathrm{C} \mathrm{NMR}\left(500 \mathrm{MHz}, \mathrm{CDCl}_{3}\right) \delta 174.0,143.9,139.2,128.3$, 127.9, 126.8, 115.4, 59.8, 58.9, 51.4, 49.7, 35.0, 32.8, 21.0, 20.9, 19.8; m/z (CI, \%) 290 $\left([\mathrm{M}+\mathrm{H}]^{+}, 40\right) 246\left(\left[\mathrm{M}-\mathrm{C}_{3} \mathrm{H}_{6}\right]^{+}, 100\right) 105\left(\mathrm{C}_{8} \mathrm{H}_{9}{ }^{+}, 30\right)$. HRMS $\mathrm{C}_{18} \mathrm{H}_{28} \mathrm{NO}_{2}$ calcd. 290.2120, found 290.2123 .

Methyl $(3 R, \alpha S)$-3-[ $N$-allyl- $N$-( $\alpha$-methylbenzyl)amino]hex-4-enoate (8c). Following general procedure $\mathrm{D}$, butyllithium in hexanes $(1.6 \mathrm{M}, 11.3 \mathrm{~mL}, 18.0 \mathrm{mmol})$ and $(E, E)$-methyl hexa2,4-dienoate $(1.47 \mathrm{~g}, 11.6 \mathrm{mmol})$ in anhydrous THF $(15 \mathrm{~mL})$ were added to $(S)-N-(\alpha$ methylbenzyl)allylamine (7) $(3.00 \mathrm{~g}, 18.6 \mathrm{mmol})$ in anhydrous THF $(15 \mathrm{~mL})$, affording an oil that was purified by flash chromatography (1:9 ethyl acetate:hexane) to give $8 \mathbf{c}(2.61 \mathrm{~g}, 78 \%)$ as a pale yellow oil; $[\alpha]_{\mathrm{D}}{ }^{21}=+3.4\left(c 2.95, \mathrm{CHCl}_{3}\right)$, lit. ${ }^{17 \mathrm{a}}$ for enantiomer $[\alpha]_{\mathrm{D}}{ }^{13}=-2.4(c 1.00$, $\left.\mathrm{CHCl}_{3}\right)$; IR $v_{\max } 2970,1736 \mathrm{~cm}^{-1} ;{ }^{1} \mathrm{H}$ NMR $\left(300 \mathrm{MHz}, \mathrm{CDCl}_{3}\right) \delta 7.35-7.17(5 \mathrm{H}, \mathrm{m}) 5.80(1 \mathrm{H}$, m) $5.51(2 \mathrm{H}, \mathrm{m}) 5.12-5.00(2 \mathrm{H}, \mathrm{m}) 4.02(1 \mathrm{H}, \mathrm{q}, J=6.8 \mathrm{~Hz}) 3.85(1 \mathrm{H}, \mathrm{m}) 3.56(3 \mathrm{H}, \mathrm{s}) 3.12$ $(2 \mathrm{H}, \mathrm{m}) 2.51(1 \mathrm{H}, \mathrm{dd}, J=14.3,5.8 \mathrm{~Hz}) 2.34(1 \mathrm{H}, \mathrm{dd}, J=14.3,7.5 \mathrm{~Hz}) 1.69(3 \mathrm{H}, \mathrm{d}, J=4.6 \mathrm{~Hz})$ $1.35(3 \mathrm{H}, \mathrm{d}, J=6.8 \mathrm{~Hz}) ;{ }^{13} \mathrm{C} \mathrm{NMR}\left(500 \mathrm{MHz}, \mathrm{CDCl}_{3}\right) \delta 172.5,145.2,138.8,130.6,128.0$, 127.6, 127.1, 126.5, 115.7, 56.9, 56.8, 51.4, 49.6, 38.9, 18.1, 18.0; (EI, \%) $288\left([\mathrm{M}+\mathrm{H}]^{+}, 20\right)$, $105\left(\mathrm{C}_{8} \mathrm{H}_{9}{ }^{+}, 100\right)$. HRMS $\mathrm{C}_{18} \mathrm{H}_{26} \mathrm{NO}_{2}$ calcd. 288.1964, found 288.1961.

Methyl (3R, $\alpha S)-3-[N$-allyl- $N$-( $\alpha$-methylbenzyl)]-3-phenylpropanoate $\quad(8 f)$. Following general procedure $\mathrm{D}$, butyllithium in hexanes $(2.5 \mathrm{M}, 3.8 \mathrm{~mL}, 9.56 \mathrm{mmol})$ and methyl $(E)$ cinnamate $(1.00 \mathrm{~g}, \quad 6.17 \mathrm{mmol})$ in anhydrous THF $(16 \mathrm{~mL})$ to $(S)-N-(\alpha-$ methylbenzyl)allylamine (7) $(1.59 \mathrm{~g}, 9.86 \mathrm{mmol})$ in anhydrous THF $(20 \mathrm{~mL})$, affording an oil that was purified by flash chromatography (10:9 ethyl acetate:hexane) to give $\mathbf{8 f}$ (1.80 g, 90\%) as a pale yellow oil; $[\alpha]_{\mathrm{D}}{ }^{21}=+1.5\left(c 4.10, \mathrm{CHCl}_{3}\right)$, lit. $^{35}$ for enantiomer $[\alpha]_{\mathrm{D}}{ }^{26}=-3.6(c$ $\left.0.85, \mathrm{CHCl}_{3}\right)$; IR $v_{\max } 2973,1736 \mathrm{~cm}^{-1} ;{ }^{1} \mathrm{H}$ NMR $\left(500 \mathrm{MHz}, \mathrm{CDCl}_{3}\right) \delta 7.55-7.33(10 \mathrm{H}, \mathrm{m})$ $5.80(1 \mathrm{H}, \mathrm{m}) 5.17(1 \mathrm{H}, \mathrm{ddd}, J=17.2,8.6,1.4 \mathrm{~Hz}) 5.08(1 \mathrm{H}, \mathrm{ddd}, J=17.2,10.1,1.4 \mathrm{~Hz}) 4.54$ $(1 \mathrm{H}, \mathrm{m}) 4.08(1 \mathrm{H}, \mathrm{q}, J=6.7 \mathrm{~Hz}) 3.57(3 \mathrm{H}, \mathrm{s}) 3.22-3.18(2 \mathrm{H}, \mathrm{m}) 2.91(1 \mathrm{H}, \mathrm{dd}, J=14.6,5.6 \mathrm{~Hz})$ $2.69(1 \mathrm{H}, \mathrm{dd}, J=14.6,8.3 \mathrm{~Hz}) 1.18(3 \mathrm{H}, \mathrm{d}, J=6.7 \mathrm{~Hz}) ;{ }^{13} \mathrm{C} \mathrm{NMR}\left(500 \mathrm{MHz}, \mathrm{CDCl}_{3}\right) \delta 172.5$, 144.9, 141.5, 138.8, 128.4, 128.1, 127.7, 127.5, 127.3, 126.7, 116.0, 58.9, 56.3, 51.5, 49.8, 38.0, 16.6; m/z (CI, \%) $324\left([\mathrm{M}+\mathrm{H}]^{+}, 18\right), 105\left(\mathrm{C}_{8} \mathrm{H}_{9}^{+}, 100\right)$. HRMS $\mathrm{C}_{21} \mathrm{H}_{26} \mathrm{NO}_{2}$ calcd. 324.1964, found 324.1958).

Methyl (3R, $\alpha S)-3-[N$-allyl- $N$-( $\alpha$-methylbenzyl)]-3-(3-pyridyl)propanoate (8g). Following general procedure D, butyllithium in hexanes $(2.5 \mathrm{M}, 1.9 \mathrm{~mL}, 4.75 \mathrm{mmol})$ and methyl 3(pyridin-3-yl)propenoate $(0.50 \mathrm{~g}, 3.06 \mathrm{mmol})$ in anhydrous THF $(5 \mathrm{~mL})(S)-N$ - $(\alpha-$ methylbenzyl)allylamine (7) $(0.95 \mathrm{~g}, 4.09 \mathrm{mmol})$ in anhydrous THF $(10 \mathrm{~mL})$ afforded an oil that was purified by flash chromatography (1:1 ethyl acetate:hexane) to give $\mathbf{8 g}(0.52 \mathrm{~g}, 48 \%)$ 
as a yellow oil; $[\alpha]_{\mathrm{D}}{ }^{21}=-0.6\left(c\right.$ 1.70, $\left.\mathrm{CHCl}_{3}\right)$; IR $v_{\max } 2972,1735 \mathrm{~cm}^{-1} ;{ }^{1} \mathrm{H} \mathrm{NMR}(500 \mathrm{MHz}$, $\left.\mathrm{CDCl}_{3}\right) \delta 8.61(1 \mathrm{H}, \mathrm{s}) 8.50(1 \mathrm{H}, \mathrm{m}) 7.67(1 \mathrm{H}, \mathrm{d}, J=7.9 \mathrm{~Hz}) 7.37-7.22(6 \mathrm{H}, \mathrm{m}) 5.78(1 \mathrm{H}, \mathrm{m})$ $5.16(2 \mathrm{H}, \mathrm{ddd}, J=17.2,4.8,1.5 \mathrm{~Hz}) 5.07(2 \mathrm{H}, \mathrm{ddd}, J=17.2,10.2,1.5 \mathrm{~Hz}) 4.54(1 \mathrm{H}, \mathrm{m}) 4.02$ $(1 \mathrm{H}, \mathrm{q}, J=6.8 \mathrm{~Hz}) 3.55(3 \mathrm{H}, \mathrm{s}) 3.19-3.13(2 \mathrm{H}, \mathrm{m}) 2.81(1 \mathrm{H}, \mathrm{dd}, J=15.2,6.0 \mathrm{~Hz}) 2.67(1 \mathrm{H}, \mathrm{dd}$, $J=15.2,9.0 \mathrm{~Hz}) 1.23(3 \mathrm{H}, \mathrm{d}, J=6.8 \mathrm{~Hz}) ;{ }^{13} \mathrm{C} \mathrm{NMR}\left(500 \mathrm{MHz}, \mathrm{CDCl}_{3}\right) \delta 172.0,149.7,148.6$, $144.2,138.2,137.2,135.3,128.3,127.5,127.0,123.3,116.4,56.9,56.6,51.7,49.8,36.7$, 17.6; $\mathrm{m} / \mathrm{z}$ (EI, \%) $325\left([\mathrm{M}+\mathrm{H}]^{+}, 15\right), 105\left(\mathrm{C}_{8} \mathrm{H}_{9}{ }^{+}, 100\right)$. HRMS $\mathrm{C}_{20} \mathrm{H}_{25} \mathrm{~N}_{2} \mathrm{O}_{2}$ calcd. 325.1916, found 325.1916 .

Methyl $\quad(3 R, \alpha S)-3-[N$-allyl- $N$-( $\alpha$-methylbenzyl)amino]-3-cyclohexylpropanoate $\quad(8 \mathrm{k})$. Following general procedure D, butyllithium in hexanes $(2.5 \mathrm{M}, 6.6 \mathrm{~mL}, 16.6 \mathrm{mmol})$ and methyl 3-cyclohexylpropenoate $(1.80 \mathrm{~g}, 10.7 \mathrm{mmol})$ in anhydrous THF $(27 \mathrm{~mL})$ were added to $(S)-N$-( $\alpha$-methylbenzyl)allylamine (7) $(2.76 \mathrm{~g}, 17.1 \mathrm{mmol})$ in anhydrous THF $(34 \mathrm{~mL})$, affording an oil that was purified by flash chromatography (12:88 ethyl acetate:hexane) to give $8 \mathbf{k}(2.40 \mathrm{~g}, 68 \%)$ as a pale yellow oil; $[\alpha]_{\mathrm{D}}{ }^{25}=+11.0\left(c 6.69, \mathrm{CHCl}_{3}\right)$; IR $v_{\max } 2923,1735$ $\mathrm{cm}^{-1}$; ${ }^{1} \mathrm{H}$ NMR $\left(500 \mathrm{MHz}, \mathrm{CDCl}_{3}\right) \delta$ 7.30-7.19 $(5 \mathrm{H}, \mathrm{m}) 5.85(1 \mathrm{H}, \mathrm{m}) 5.20(1 \mathrm{H}, \mathrm{m}) 5.07(1 \mathrm{H}$, m) $3.90(1 \mathrm{H}, \mathrm{q}, J=7.0 \mathrm{~Hz}) 3.57(3 \mathrm{H}, \mathrm{s}) 3.17-3.12(2 \mathrm{H}, \mathrm{m}) 3.06(1 \mathrm{H}, \mathrm{m}) 2.17-2.08(2 \mathrm{H}, \mathrm{m})$ $2.00(1 \mathrm{H}, \mathrm{dd}, J=15.7,3.7 \mathrm{~Hz}) 1.57-1.42(4 \mathrm{H}, \mathrm{m}) 1.39(3 \mathrm{H}, \mathrm{d}, J=7.0 \mathrm{~Hz}) 1.17-1.12(4 \mathrm{H}, \mathrm{m})$ 0.88-0.76 (2H, m); $\left.{ }^{13} \mathrm{C} \mathrm{NMR} \mathrm{(500} \mathrm{MHz,} \mathrm{CDCl}_{3}\right) \delta 174.0,143.9,139.2,128.3,127.9,126.8$, $115.4,58.8,51.4,49.8,42.7,34.7,31.1,30.2,27.0,26.6,21.0 ; \mathrm{m} / \mathrm{z}(\mathrm{CI}, \%) 330\left([\mathrm{M}+\mathrm{H}]^{+}, 20\right)$ $246\left(\left[\mathrm{M}-\mathrm{C}_{7} \mathrm{H}_{10}\right]^{+}, 100\right)$. HRMS $\mathrm{C}_{21} \mathrm{H}_{32} \mathrm{NO}_{2}$ calcd. 330.2433, found 330.2439.

Methyl (3S, $\alpha S)-3-[N$-( $\alpha$-methylbenzyl)]-butanoate (9a). Following general procedure E, reaction of amine $8 \mathbf{a}(1.00 \mathrm{~g}, 3.83 \mathrm{mmol})$ and Wilkinson's catalyst $(180 \mathrm{mg}, 0.19 \mathrm{mmol})$ in acetonitrile-water $(85: 15,20 \mathrm{~mL})$ at $20{ }^{\circ} \mathrm{C}$ afforded an oil that was purified by flash chromatography (15:85 ethyl acetate:hexane) to give 9a $(0.73 \mathrm{~g}, 87 \%)$ as a pale brown oil; $[\alpha]_{\mathrm{D}}{ }^{21}=-22.5\left(c 1.29, \mathrm{CHCl}_{3}\right)$; IR $v_{\max }\left(\mathrm{cm}^{-1}\right) 2960,2923,1731 \mathrm{~cm}^{-1} ;{ }^{1} \mathrm{H}$ NMR $(500 \mathrm{MHz}$,

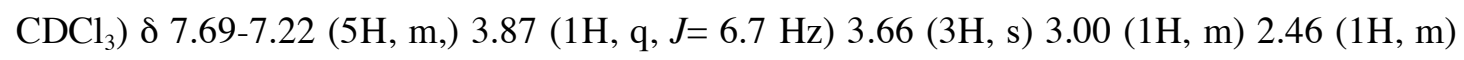
$2.37(1 \mathrm{H}, \mathrm{m}) 1.68(1 \mathrm{H}, \mathrm{br} \mathrm{s}) 1.33(3 \mathrm{H}, \mathrm{d}, J=6.7 \mathrm{~Hz}) 1.05(3 \mathrm{H}, \mathrm{d}, J=6.7 \mathrm{~Hz}) ;{ }^{13} \mathrm{C}$ NMR $(500$ $\left.\mathrm{MHz}, \mathrm{CDCl}_{3}\right) \delta 172.8,132.0,128.6,127.0,126.6,55.3,51.5,47.8,40.6,24.6,21.5 ; \mathrm{m} / \mathrm{z}(\mathrm{CI}$, \%) $222\left([\mathrm{M}+\mathrm{H}]^{+}, 100\right)$. HRMS $\mathrm{C}_{13} \mathrm{H}_{20} \mathrm{NO}_{2}$ calcd. 222.1494, found 292.1491.

Methyl (3R, $\alpha S)-3-[N$-( $\alpha$-methylbenzyl)]-4-methylpentanoate $(9 b)$. Following general procedure E, reaction of amine $\mathbf{8 b}(1.50 \mathrm{~g}, 5.18 \mathrm{mmol})$ and Wilkinson's catalyst $(0.24 \mathrm{~g}, 0.26$ mmol) in acetonitrile-water $(85: 15,30 \mathrm{~mL})$ at $20^{\circ} \mathrm{C}$ afforded an oil that was purified by flash chromatography (18:82 ethyl acetate:hexane) to give $\mathbf{9 b}(0.77 \mathrm{~g}, 59 \%)$ as a pale yellow oil; $[\alpha]_{\mathrm{D}}^{21}=-48.1\left(c 2.35, \mathrm{CHCl}_{3}\right)$; IR $v_{\max } 2957,1733 \mathrm{~cm}^{-1} ;{ }^{1} \mathrm{H} \mathrm{NMR}\left(500 \mathrm{MHz}, \mathrm{CDCl}_{3}\right) \delta 7.38-$ 
$7.20(5 \mathrm{H}, \mathrm{m}) 3.84(1 \mathrm{H}, \mathrm{m}) 3.67(3 \mathrm{H}, \mathrm{s}) 2.65(1 \mathrm{H}, \mathrm{q}, J=6.3 \mathrm{~Hz}) 2.45(1 \mathrm{H}, \mathrm{dd}, J=14.6,9.0 \mathrm{~Hz})$ $2.37(1 \mathrm{H}, \mathrm{dd}, J=14.6,5.5 \mathrm{~Hz}) 1.67(1 \mathrm{H}, \mathrm{m}) 1.31(3 \mathrm{H}, \mathrm{d}, J=6.3 \mathrm{~Hz}) 0.87(3 \mathrm{H}, \mathrm{d}, J=6.8 \mathrm{~Hz})$ $0.80(3 \mathrm{H}, \mathrm{d}, J=6.8 \mathrm{~Hz}) ;{ }^{13} \mathrm{C}$ NMR $\left(500 \mathrm{MHz}, \mathrm{CDCl}_{3}\right) \delta 173.5,132.0,128.3,126.9,126.5$, 57.6, 55.5, 51.5, 35.9, 31.4, 24.9, 18.6, 18.5; m/z (CI, \%) $250\left([\mathrm{M}+\mathrm{H}]^{+}, 100\right) 206\left(\left[\mathrm{M}_{-} \mathrm{C}_{3} \mathrm{H}_{6}\right]^{+}\right.$, 53). HRMS $\mathrm{C}_{15} \mathrm{H}_{24} \mathrm{NO}_{2}$ calcd. 250.1807, found 250.1805 .

(4E)-Methyl $\quad(3 R, \alpha S)-3-[N$-( $\alpha$-methylbenzyl) $]$ hex-4-enoate $\quad(9 \mathrm{c}) . \quad$ Following general procedure E, reaction of amine $8 \mathbf{c}(5.10 \mathrm{~g}, 17.8 \mathrm{mmol})$ and Wilkinson's catalyst $(0.82 \mathrm{~g}, 0.89$ mmol) in acetonitrile-water $(85: 15,120 \mathrm{~mL})$ at $20^{\circ} \mathrm{C}$ afforded an oil that was purified by flash chromatography (1:9 ethyl acetate:hexane) to give $9 \mathrm{c}(3.69 \mathrm{~g}, 84 \%)$ as a pale yellow oil; $[\alpha]_{\mathrm{D}}^{21}=-38.9\left(c 0.72, \mathrm{CHCl}_{3}\right) ; \mathrm{IR} v_{\max } 3060,2965,1734 \mathrm{~cm}^{-1} ;{ }^{1} \mathrm{H} \mathrm{NMR}\left(300 \mathrm{MHz}, \mathrm{CDCl}_{3}\right) \delta$ 7.57-7.04 $(5 \mathrm{H}, \mathrm{m}) 5.55(1 \mathrm{H}, \mathrm{m}) 5.27(1 \mathrm{H}, \mathrm{m}) 3.83(1 \mathrm{H}, \mathrm{q}, J=6.5 \mathrm{~Hz}) 3.66(3 \mathrm{H}, \mathrm{s}) 3.48(1 \mathrm{H}$, m) $2.52-2.46(2 \mathrm{H}, \mathrm{m}) 1.63(3 \mathrm{H}, \mathrm{dd}, J=6.5,1.4 \mathrm{~Hz}) 1.31(3 \mathrm{H}, \mathrm{d}, J=6.5 \mathrm{~Hz}) ;{ }^{13} \mathrm{C}$ NMR $(500$ $\left.\mathrm{MHz}, \mathrm{CDCl}_{3}\right) \delta 172.5,146.2,132.7,128.5,127.1,126.9,126.7,54.9,54.7,51.5,40.5,23.3$, 17.8; (EI, \%) $247\left([\mathrm{M}+\mathrm{H}]^{+}, 5\right) 232\left(\left[\mathrm{M}-\mathrm{CH}_{3}\right]^{+}, 38\right), 105\left(\mathrm{C}_{8} \mathrm{H}_{9}{ }^{+}, 100\right)$. HRMS $\mathrm{C}_{15} \mathrm{H}_{22} \mathrm{NO}_{2}$ calcd. 248.1650, found 248.1651.

Methyl (3R, $\alpha S)-3$-[N-( $\alpha$-methylbenzyl)]-3-phenylpropanoate (9f). Following general procedure E, reaction of amine $\mathbf{8 f}(0.50 \mathrm{~g}, 1.55 \mathrm{mmol})$ and Wilkinson's catalyst $(72 \mathrm{mg}, 0.08$ mmol) in acetonitrile-water $(85: 15,10 \mathrm{~mL})$ at $20{ }^{\circ} \mathrm{C}$ gave an oil that was purified by flash chromatography (1:4 ethyl acetate:hexane) to give 9f $(0.33 \mathrm{~g}, 76 \%)$ as a pale yellow oil; $[\alpha]_{\mathrm{D}}^{21}=-13.5\left(c 7.47, \mathrm{CHCl}_{3}\right)$, lit. $^{36}[\alpha]_{\mathrm{D}}^{20}=-16.3\left(c 1.00, \mathrm{CHCl}_{3}\right)$; IR $v_{\max } 3050,2964,1732$ $\mathrm{cm}^{-1} ;{ }^{1} \mathrm{H}$ NMR $\left(500 \mathrm{MHz}, \mathrm{CDCl}_{3}\right) \delta$ 7.33-7.21 $(10 \mathrm{H}, \mathrm{m}) 4.21(1 \mathrm{H}, \mathrm{m}) 3.66(1 \mathrm{H}, \mathrm{q}, J=6.6 \mathrm{~Hz})$ $3.62(3 \mathrm{H}, \mathrm{s}) 2.77(1 \mathrm{H}, \mathrm{m}) 2.68(1 \mathrm{H}, \mathrm{m}) 1.85(1 \mathrm{H}, \mathrm{br} \mathrm{s}) 1.23(3 \mathrm{H}, \mathrm{d}, J=6.6 \mathrm{~Hz}) ;{ }^{13} \mathrm{C}$ NMR $(500$ $\left.\mathrm{MHz}, \mathrm{CDCl}_{3}\right) \delta 172.2,132.0,128.6,128.5,127.5,127.1,126.7,56.9,54.8,51.6,42.5,22.2$; $m / z(\mathrm{CI}, \%) 284\left([\mathrm{M}+\mathrm{H}]^{+}, 100\right)$. HRMS $\mathrm{C}_{18} \mathrm{H}_{22} \mathrm{NO}_{2}$ calcd. 284.1651, found 284.1648.

\section{Methyl $\quad(3 R, \alpha S)-3-[N-(\alpha-$ methylbenzyl)]-3-(pyridin-3-yl)propanoate}

$(9 \mathrm{~g})$.

Tetrakis(triphenylphosphine)palladium(0) $(0.16 \mathrm{~g}, 0.01 \mathrm{mmol})$ and dimethylbarbituric acid $(0.73 \mathrm{~g}, 2.98 \mathrm{mmol})$ were added to a stirred solution of amine $8 \mathrm{~g}(0.54 \mathrm{~g}, 1.51 \mathrm{mmol})$ in anhydrous dichloromethane $(80 \mathrm{~mL})$ at $30{ }^{\circ} \mathrm{C}$ under nitrogen. After stirring the solution at 30 ${ }^{\circ} \mathrm{C}$ for a further $3 \mathrm{~h}$ the mixture was washed with aqueous saturated sodium carbonate $(2 \mathrm{x} 10$ $\mathrm{mL})$, and the combined organic layers dried $\left(\mathrm{MgSO}_{4}\right)$ and evaporated to give an oil that was purified by flash chromatography (6:4 ethyl acetate:hexane) to give $9 \mathrm{~g}(0.41 \mathrm{~g}, 96 \%)$ as an orange oil; IR $v_{\max } 2982,2920,1732 \mathrm{~cm}^{-1} ;{ }^{1} \mathrm{H}$ NMR $\left(500 \mathrm{MHz}, \mathrm{CDCl}_{3}\right) \delta 8.47(1 \mathrm{H}, \mathrm{s}) 8.40$ $(1 \mathrm{H}, \mathrm{m}) 7.65(1 \mathrm{H}, \mathrm{m}) 7.43-7.14(6 \mathrm{H}, \mathrm{m}) 4.17(1 \mathrm{H}, \mathrm{dd}, J=7.5,6.3 \mathrm{~Hz}) 3.65(1 \mathrm{H}, \mathrm{q}, J=6.4 \mathrm{~Hz})$ $3.58(3 \mathrm{H}, \mathrm{s}) 2.75(1 \mathrm{H}, \mathrm{dd}, J=15.5,7.5 \mathrm{~Hz}) 2.63(1 \mathrm{H}, \mathrm{dd}, J=15.5,6.3 \mathrm{~Hz}) 2.25(1 \mathrm{H}, \mathrm{br} \mathrm{s}, \mathrm{NH})$ 
$1.33(3 \mathrm{H}, \mathrm{d}, J=6.4 \mathrm{~Hz}) ;{ }^{13} \mathrm{C}$ NMR $\left(500 \mathrm{MHz}, \mathrm{CDCl}_{3}\right) \delta 171.7,149.3,148.8,145.4,138.2$, 133.7, 132.0, 128.9, 128.5, 127.1, 55.4, 54.9, 51.7, 41.8, 22.9; $\mathrm{m} / \mathrm{z}(\mathrm{CI}, \%) 285\left([\mathrm{M}+\mathrm{H}]^{+}\right.$, 100). HRMS $\mathrm{C}_{17} \mathrm{H}_{21} \mathrm{~N}_{2} \mathrm{O}_{2}$ calcd. 285.1603, found 285.1607.

Methyl (3R, $\alpha S)-3-[N$-( $\alpha$-methylbenzyl)]-3-cyclohexylpropanoate (9k). Following general procedure E, reaction of amine $\mathbf{8 k}(1.86 \mathrm{~g}, 5.65 \mathrm{mmol})$ and Wilkinson's catalyst $(0.30 \mathrm{~g}, 0.32$ $\mathrm{mmol})$ in $85: 15$ acetonitrile:water $(35 \mathrm{~mL})$ at $20^{\circ} \mathrm{C}$ afforded an oil that was purified by flash chromatography (12:88 ethyl acetate:hexane) to give $9 \mathbf{k}(0.79 \mathrm{~g}, 53 \%)$ as a yellow oil; $[\alpha]_{\mathrm{D}}{ }^{25}$ $=-35.2\left(c 1.90, \mathrm{CHCl}_{3}\right)$; IR $v_{\max } 2985,2922,1732 \mathrm{~cm}^{-1} ;{ }^{1} \mathrm{H}$ NMR $\left(500 \mathrm{MHz}, \mathrm{CDCl}_{3}\right) \delta 7.35-$ $7.20(5 \mathrm{H}, \mathrm{m}) 3.80(1 \mathrm{H}, \mathrm{m}) 3.67(3 \mathrm{H}, \mathrm{s}) 2.63(1 \mathrm{H}, \mathrm{q}, J=5.9 \mathrm{~Hz}) 2.49(1 \mathrm{H}, \mathrm{dd}, J=14.7,5.5 \mathrm{~Hz})$ $2.37(1 \mathrm{H}, \mathrm{dd}, J=14.7,6.3 \mathrm{~Hz}) 1.79-1.62(5 \mathrm{H}, \mathrm{m}) 1.31(3 \mathrm{H}, \mathrm{d}, J=5.9 \mathrm{~Hz}) 1.17-1.08(4 \mathrm{H}, \mathrm{m})$ 0.96-0.86 (2H, m); ${ }^{13} \mathrm{C}$ NMR (500 MHz, $\left.\mathrm{CDCl}_{3}\right) \delta 173.5,132.0,128.3,127.0,126.9,57.0$, $55.5,51.5,41.8,35.9,29.3,29.1,26.6,26.5 ; \mathrm{m} / \mathrm{z}(\mathrm{CI}, \%) 290\left([\mathrm{M}+\mathrm{H}]^{+}, 100\right)$. HRMS $\mathrm{C}_{18} \mathrm{H}_{28} \mathrm{NO}_{2}$ calcd. 290.2120, found 290.2112.

Methyl (3S, $\alpha S)-3-[N$-( $\alpha$-methylbenzyl), $N$-(2-methoxycarbonylacetyl)]butanoate (10a). Following general procedure B, reaction of amine 9a $(0.30 \mathrm{~g}, 1.36 \mathrm{mmol})$, triethylamine $(0.22$ $\mathrm{mL}, 1.63 \mathrm{mmol})$ and methyl 3-chloro-3-oxopropanoate $(0.16 \mathrm{~mL}, 1.5 \mathrm{mmol})$ in dichloromethane $(6 \mathrm{~mL})$ afforded an oil that was purified by flash chromatography (1:1 ethyl acetate:hexane) to give $\mathbf{1 0 a}(0.25 \mathrm{~g}, 58 \%)$ as a colourless oil; $[\alpha]_{\mathrm{D}}{ }^{21}=-2.5\left(c 0.80, \mathrm{CHCl}_{3}\right)$; IR $v_{\max } 2952,1736,1646 \mathrm{~cm}^{-1} ;{ }^{1} \mathrm{H}$ NMR $\left(500 \mathrm{MHz}, \mathrm{CDCl}_{3}\right) \delta 7.38-7.22(5 \mathrm{H}, \mathrm{m}) 4.95(1 \mathrm{H}, \mathrm{q}, J=$ $6.8 \mathrm{~Hz}) 3.78(3 \mathrm{H}, \mathrm{s}) 3.60(3 \mathrm{H}, \mathrm{s}) 3.46(2 \mathrm{H}, \mathrm{s}) 3.08(1 \mathrm{H}, \mathrm{m}) 2.42(1 \mathrm{H}, \mathrm{m}) 2.08(1 \mathrm{H}, \mathrm{m}) 1.66$ $(3 \mathrm{H}, \mathrm{d}, J=6.8 \mathrm{~Hz}) 1.43(3 \mathrm{H}, \mathrm{d}, J=6.7 \mathrm{~Hz}) ;{ }^{13} \mathrm{C} \mathrm{NMR}\left(500 \mathrm{MHz}, \mathrm{CDCl}_{3}\right) \delta 172.3,168.3$, 165.6, 139.0, 128.3, 128.1, 127.3, 56.9, 52.6, 51.4, 48.6, 42.9, 39.1, 18.7, 17.3; m/z (CI, \%) $321\left(\mathrm{M}^{+}, 5\right) 220\left(\left[\mathrm{M}-\mathrm{C}_{4} \mathrm{H}_{5} \mathrm{O}_{3}\right]^{+}, 85\right) 105\left(\mathrm{C}_{8} \mathrm{H}_{9}{ }^{+}, 100\right)$. HRMS $\mathrm{C}_{17} \mathrm{H}_{23} \mathrm{NO}_{5}$ calcd. 321.1576, found 321.1571 .

\section{Methyl $\quad(3 R, \alpha S)-3-[N$-( $\alpha$-methylbenzyl)], $\quad N$-(2-methoxycarbonylacetyl)]-4-}

methylpentanoate (10b). Following general procedure B, reaction of amine $9 \mathbf{b}(0.25 \mathrm{~g}, 1.0$ $\mathrm{mmol})$, triethylamine $(0.18 \mathrm{~mL}, 1.30 \mathrm{mmol})$ and methyl 3-chloro-3-oxopropanoate $(0.13 \mathrm{~mL}$, $1.2 \mathrm{mmol})$ in dichloromethane $(4 \mathrm{~mL})$ afforded a pale yellow oil that was purified by flash chromatography (1:1 ethyl acetate:hexane) to give $\mathbf{1 0 b}(0.18 \mathrm{~g}, 50 \%)$ as a pale yellow oil; $[\alpha]_{\mathrm{D}}{ }^{21}=+74.3\left(c 0.35, \mathrm{CHCl}_{3}\right) ; \mathrm{IR} v_{\max } 2953,1738,1647 \mathrm{~cm}^{-1} ;{ }^{1} \mathrm{H} \mathrm{NMR}\left(500 \mathrm{MHz}, \mathrm{CDCl}_{3}\right)$ (presence of rotamers) $\delta$ 7.35-7.22 $(5 \mathrm{H}, \mathrm{m}) 5.00(1 \mathrm{H}, \mathrm{m}) 3.95(1 \mathrm{H}, \mathrm{d}, J=15.7 \mathrm{~Hz}$, rotamer A) $3.78(3 \mathrm{H}, \mathrm{s}) 3.72(1 \mathrm{H}, \mathrm{d}, J=15.2 \mathrm{~Hz}$, rotamer B) $3.68(1 \mathrm{H}, \mathrm{d}, J=15.7 \mathrm{~Hz}$, rotamer A) 3.60 $(1 \mathrm{H}, \mathrm{d}, J=15.2 \mathrm{~Hz}$, rotamer B) 3.46 and $3.36(3 \mathrm{H}, \mathrm{s}) 3.27(1 \mathrm{H}, \mathrm{m}) 2.72(1 \mathrm{H}, \mathrm{dd}, J=18.0,6.9$ Hz) $2.24(1 \mathrm{H}, \mathrm{dd}, J=18.0,2.5 \mathrm{~Hz}) 2.04(1 \mathrm{H}, \mathrm{m}) 1.88$ and $1.65(3 \mathrm{H}, \mathrm{d}, J=7.0 \mathrm{~Hz}) 1.11$ and 
$0.99(3 \mathrm{H}, \mathrm{d}, J=6.7 \mathrm{~Hz}) 0.92$ and $0.81(3 \mathrm{H}, \mathrm{d}, J=6.8 \mathrm{~Hz}) ;{ }^{13} \mathrm{C} \mathrm{NMR}\left(500 \mathrm{MHz}, \mathrm{CDCl}_{3}\right) \delta$ $172.7,171.9,168.6,168.4,167.6,165.9,139.2,128.8,128.3,127.9,127.7,126.8,126.6$, 62.3, 58.3, 56.6, 55.6, 52.6, 52.4, 51.9, 51.4, 43.0, 42.8, 39.6, 35.9, 31.2, 30.9, 21.4, 21.2, 20.7, 20.1, 19.9, 18.3; m/z (CI, \%) $372\left(\left[\mathrm{M}+\mathrm{Na}^{+}\right], 100\right)$. HRMS $\mathrm{C}_{19} \mathrm{H}_{27} \mathrm{NO}_{5} \mathrm{Na}$ calcd. 372.1787, found 372.1783 .

(4E)-Methyl $(3 R, \alpha S)-3$-[ $N$-( $\alpha$-methylbenzyl)], $N$-(2-methoxycarbonylacetyl)]hex-4-enoate (10c). Following general procedure B, reaction of amine $9 \mathrm{c}(1.2 \mathrm{~g}, 4.85 \mathrm{mmol})$, triethylamine $(0.80 \mathrm{~mL}, 5.8 \mathrm{mmol})$ and methyl 3-chloro-3-oxopropanoate $(0.57 \mathrm{~mL}, 5.3 \mathrm{mmol})$ in dichloromethane $(25 \mathrm{~mL})$ afforded an oil that was purified by flash chromatography (3:7 ethyl acetate:hexane) to give $10 \mathrm{c}(1.58 \mathrm{~g}, 94 \%)$ as a colourless oil; $[\alpha]_{\mathrm{D}}{ }^{21}=+5.5\left(\mathrm{c} 1.82, \mathrm{CHCl}_{3}\right)$; IR $v_{\max } 3030(\mathrm{~N}-\mathrm{H}), 2952,1733,1641 \mathrm{~cm}^{-1} ;{ }^{1} \mathrm{H}$ NMR (300 MHz, $\left.\mathrm{CDCl}_{3}\right) \delta$ 7.38-7.21 (5H, m) $5.93(1 \mathrm{H}, \mathrm{m}) 5.65-5.56(2 \mathrm{H}, \mathrm{m}) 5.04(1 \mathrm{H}, \mathrm{q}, J=6.7 \mathrm{~Hz}) 3.78(3 \mathrm{H}, \mathrm{s}) 3.51(2 \mathrm{H}, \mathrm{s}) 3.45(3 \mathrm{H}, \mathrm{s})$ $1.74(2 \mathrm{H}, \mathrm{d}, J=4.9 \mathrm{~Hz}) 1.64(3 \mathrm{H}, \mathrm{d}, J=6.7 \mathrm{~Hz}) 1.58(3 \mathrm{H}, \mathrm{m}) ; \mathrm{m} / z(\mathrm{CI}, \%) 370\left(\mathrm{M}^{+}, 100\right), 311$ ([M-CO $\left.\left.\mathrm{CO}_{2} \mathrm{Me}\right]^{+}, 2\right)$. HRMS $\mathrm{C}_{19} \mathrm{H}_{25} \mathrm{NO}_{5} \mathrm{Na}$ calcd. 370.1630, found 370.1626 .

Methyl $\quad(3 R, \alpha S)-3-[N$-( $\alpha$-methylbenzyl), $\quad N$-(2-methoxycarbonylacetyl)]-3phenylpropanoate (10f). Following general procedure B, reaction of amine 9f (4.16 g, 14.7 $\mathrm{mmol})$, triethylamine $(2.7 \mathrm{~mL}, 19 \mathrm{mmol})$ and methyl 3-chloro-3-oxopropanoate $(1.9 \mathrm{~mL}, 17.6$ $\mathrm{mmol})$ in dichloromethane $(45 \mathrm{~mL})$ afforded an oil that was purified by flash chromatography (35:65 ethyl acetate:hexane) to give $\mathbf{1 0 f}(4.48 \mathrm{~g}, 80 \%)$ as a colourless oil; $[\alpha]_{\mathrm{D}}{ }^{21}=+25.0(\mathrm{c}$ 1.56, $\mathrm{CHCl}_{3}$ ); IR $v_{\max } 2950,1735,1647 \mathrm{~cm}^{-1} ;{ }^{1} \mathrm{H} \mathrm{NMR}\left(300 \mathrm{MHz}, \mathrm{CDCl}_{3}\right.$ ) (presence of rotamers) $\delta$ 7.37-7.17 $(10 \mathrm{H}, \mathrm{m}) 5.32$ and $5.24(1 \mathrm{H}, \mathrm{m}) 4.98(1 \mathrm{H}, \mathrm{q}, J=6.1 \mathrm{~Hz}) 3.71(3 \mathrm{H}, \mathrm{s})$ $3.56(2 \mathrm{H}, \mathrm{s}) 3.51(3 \mathrm{H}, \mathrm{s}) 3.36-3.31(2 \mathrm{H}, \mathrm{m}) 2.96(1 \mathrm{H}, \mathrm{m}) 2.73-2.65(1 \mathrm{H}, \mathrm{m}) 1.25(3 \mathrm{H}, J=6.1$ $\mathrm{Hz}) ;{ }^{13} \mathrm{C}$ NMR (500 MHz, $\mathrm{CDCl}_{3}$ ) $\delta$ 171.9, 171.1, 168.4, 168.2, 167.2, 166.5, 141.4, 140.4, 139.9, 139.0, 129.1, 129.0, 128.6, 128.5, 128.2, 128.0, 127.4, 127.2 127.0, 126.6, 126.5, 56.8, 56.4, 55.2, 54.7, 52.6, 52.5, 52.1, 51.8, 43.0, 40.8, 38.9, $38.318 .6 ; \mathrm{m} / z(\mathrm{CI}, \%) 384\left([\mathrm{M}-\mathrm{H}]^{+}\right.$, 100). HRMS $\mathrm{C}_{22} \mathrm{H}_{26} \mathrm{NO}_{5} \mathrm{Na}$ calcd. 384.1811, found 384.1803 .

Methyl $\quad(3 R, \alpha S)-3-[N$-( $\alpha$-methylbenzyl), $\quad N$-(2-methoxycarbonylacetyl)]-3-(pyridin-3yl)propanoate $(\mathbf{1 0 g})$. Following general procedure B, reaction of amine 9f $(0.16 \mathrm{~g}, 0.56$ $\mathrm{mmol})$, triethylamine $(0.11 \mathrm{~mL}, 0.73 \mathrm{mmol})$ and methyl 3-chloro-3-oxopropanoate $(0.07 \mathrm{~mL}$, $0.67 \mathrm{mmol})$ in dichloromethane $(3 \mathrm{~mL})$ afforded an oil that was purified by flash chromatography (1:1 ethyl acetate:hexane) to give $10 \mathrm{~g}(0.16 \mathrm{~g}, 75 \%)$ as a colourless oil; $[\alpha]_{\mathrm{D}}{ }^{21}=+15.0\left(c 1.00, \mathrm{CHCl}_{3}\right)$; IR $v_{\max } 2952,1735,16481496 \mathrm{~cm}^{-1} ;{ }^{1} \mathrm{H}$ NMR $(500 \mathrm{MHz}$, $\mathrm{CDCl}_{3}$ ) (presence of rotamers) $\delta 8.57(1 \mathrm{H}, \mathrm{s}) 8.40(1 \mathrm{H}, \mathrm{m}) 7.82(1 \mathrm{H}, \mathrm{d}, J=7.3 \mathrm{~Hz}) 7.64(1 \mathrm{H}$, m) 7.45-7.17 (5H, m) $5.08(1 \mathrm{H}, \mathrm{q}, J=6.5 \mathrm{~Hz}) 4.86(1 \mathrm{H}, \mathrm{dd}, J=7.7,5.6 \mathrm{~Hz}) 3.74(3 \mathrm{H}, \mathrm{s}) 3.58$ 
$(2 \mathrm{H}, \mathrm{s}) 3.43(3 \mathrm{H}, \mathrm{s}) 2.60(1 \mathrm{H}, \mathrm{dd}, J=17.1,7.7 \mathrm{~Hz}) 2.27(1 \mathrm{H}, \mathrm{dd}, J=17.1,5.6 \mathrm{~Hz}) 1.60(3 \mathrm{H}, \mathrm{d}$, $J=6.5 \mathrm{~Hz}) ;{ }^{13} \mathrm{C} \mathrm{NMR}\left(500 \mathrm{MHz}, \mathrm{CDCl}_{3}\right) \delta 171.6,168.1,166.2,149.0,148.5,136.1,135.7$, 132.1, 132.0, 129.1, 128.8, 128.4, 127.2, 123.3, 57.3, 53.3, 52.6, 51.8, 42.7, 38.7, 18.4; m/z $(\mathrm{CI}, \%) 385\left([\mathrm{M}+\mathrm{H}]^{+}, 100\right) 279\left(\left[\mathrm{M}-\mathrm{C}_{8} \mathrm{H}_{8}\right]^{+}, 55\right)$. HRMS $\mathrm{C}_{21} \mathrm{H}_{25} \mathrm{~N}_{2} \mathrm{O}_{5}$ calcd. 385.1764, found 385.1761 .

Methyl $\quad(3 R, \alpha S)-3-[N$-( $\alpha$-methylbenzyl), $\quad N$-(2-methoxycarbonylacetyl)]-3cyclohexylpropanoate $(\mathbf{1 0 k})$. Following general procedure B, reaction of amine $9 \mathbf{k}(0.25 \mathrm{~g}$, $0.86 \mathrm{mmol})$, triethylamine $(0.16 \mathrm{~mL}, 1.12 \mathrm{mmol})$ and methyl 3-chloro-3-oxopropanoate $(0.11$ $\mathrm{mL}, 1.04 \mathrm{mmol})$ in dichloromethane $(4 \mathrm{~mL})$ afforded a pale yellow oil that was purified by flash chromatography (1:1 ethyl acetate:hexane) to give 10k $(0.22 \mathrm{~g}, 66 \%)$ as a pale yellow oil, $[\alpha]_{\mathrm{D}}^{25}=+22.5\left(c 1.00, \mathrm{CHCl}_{3}\right)$; IR $v_{\max } 2925,1737,1648 \mathrm{~cm}^{-1} ;{ }^{1} \mathrm{H}$ NMR $(500 \mathrm{MHz}$, $\mathrm{CDCl}_{3}$ ) (presence of rotamers) $\delta$ 7.32-7.16 $(5 \mathrm{H}, \mathrm{m}) 4.99-4.95$ and $4.30-4.28(1 \mathrm{H}, \mathrm{m}) 3.77$ $(3 \mathrm{H}, \mathrm{s}) 3.65(1 \mathrm{H}, \mathrm{s}) 3.60(1 \mathrm{H}, \mathrm{s}) 3.40(3 \mathrm{H}, \mathrm{s}) 2.72$ and $2.46(1 \mathrm{H}, \mathrm{d}, J=7.0 \mathrm{~Hz}) 2.45-2.02(2 \mathrm{H}$, m) $1.84(3 \mathrm{H}, \mathrm{d}, J=7.0 \mathrm{~Hz}) 1.73-1.61(5 \mathrm{H}, \mathrm{m}) 1.29-0.82(6 \mathrm{H}, \mathrm{m}) ;{ }^{13} \mathrm{C} \mathrm{NMR}\left(500 \mathrm{MHz}, \mathrm{CDCl}_{3}\right)$

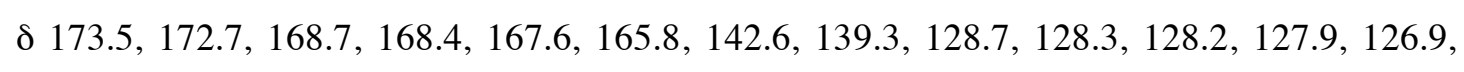
126.8, 61.4, 57.2 , 57.0, 55.5, 52.5, 51.5, 51.3, 50.0, 43.0, 42.8 41.8, 40.8, 35.9, 31.5, 30.6, $30.1,29.2,29.1,26.6,26.5,26.4,26.3,26.1,26.0,25.0,21.2 ; \mathrm{m} / \mathrm{z}(\mathrm{CI}, \%) 412\left([\mathrm{M}+\mathrm{Na}]^{+}\right.$, 100). HRMS $\mathrm{C}_{22} \mathrm{H}_{31} \mathrm{NO}_{5} \mathrm{Na}$ calcd. 412.2100, found 412.2115 .

(1S,6R)-1-( $\alpha$-Methylbenzyl)-3-methoxycarbonyl-6-phenylpiperidine-2,4-dione sodium salt (11f). Sodium methoxide in methanol $(1.97 \mathrm{M}, 0.2 \mathrm{~mL}, 0.38 \mathrm{mmol})$ was added to a stirred solution of diester $10 \mathrm{f}(0.13 \mathrm{~g}, 0.34 \mathrm{mmol})$ in methanol $(0.70 \mathrm{~mL})$ at $20^{\circ} \mathrm{C}$, under nitrogen. The mixture was then heated under reflux for $1 \mathrm{~h}$, allowed to cool to $20^{\circ} \mathrm{C}$, then diluted with diethyl ether and filtered to give $11 \mathbf{f}(0.12 \mathrm{~g}, 97 \%)$ as a white solid, $\mathrm{mp} 230{ }^{\circ} \mathrm{C}$ (decomp.); IR $v_{\max } 3220,2972,1671,16501589 \mathrm{~cm}^{-1}$; ${ }^{1} \mathrm{H}$ NMR $\left(500 \mathrm{MHz}, \mathrm{CDCl}_{3}\right) \delta 7.38$ $7.14(10 \mathrm{H}, \mathrm{m}) 6.21(1 \mathrm{H}, \mathrm{q}, J=7.2 \mathrm{~Hz}) 4.33(1 \mathrm{H}, \mathrm{dd}, J=6.9,1.3 \mathrm{~Hz}) 3.67(3 \mathrm{H}, \mathrm{s}) 2.64(1 \mathrm{H}, \mathrm{dd}$, $J=15.7,6.9 \mathrm{~Hz}) 2.15(1 \mathrm{H}, \mathrm{dd}, J=15.7,1.3 \mathrm{~Hz}) 1.24(3 \mathrm{H}, \mathrm{d}, J=7.2 \mathrm{~Hz}) ;{ }^{13} \mathrm{C}$ NMR $(500 \mathrm{MHz}$, $\left.\mathrm{CDCl}_{3}\right) \delta 186.6,171.8,170.6,144.1,143.9,129.5,129.2,128.3,128.1,127.9,127.7,97.5$, 53.6, 51.4, 50.5, 44.9, 17.0.

\section{(1S,6R)-1-( $\alpha$-Methylbenzyl)-3-methoxycarbonyl-6-(E)-propenylpiperidine-2,4-dione}

sodium salt (11). Sodium methoxide in methanol (2.14 M, $2.3 \mathrm{~mL}, 5.0 \mathrm{mmol})$ was added to a stirred solution of diester $101(1.58 \mathrm{~g}, 4.6 \mathrm{mmol})$ in methanol $(6 \mathrm{~mL})$ at $20{ }^{\circ} \mathrm{C}$, under nitrogen. The mixture was then heated under reflux for $1 \mathrm{~h}$. After allowing to cool to $20^{\circ} \mathrm{C}$, the mixture was diluted with diethyl ether and filtered to give $\mathbf{1 1 1}(1.46 \mathrm{~g}, 95 \%)$ as a white solid, mp 203-207 ${ }^{\circ} \mathrm{C}$; IR $v_{\max } 3064,2974,1660 \mathrm{~cm}^{-1}$; ${ }^{1} \mathrm{H}$ NMR $\left(500 \mathrm{MHz}, \mathrm{CDCl}_{3}\right) \delta$ 7.35- 
$7.22(5 \mathrm{H}, \mathrm{m}) 6.04(1 \mathrm{H}, \mathrm{q}, J=7.2 \mathrm{~Hz}) 5.66(1 \mathrm{H}, \mathrm{m}) 5.53(1 \mathrm{H}, \mathrm{m}) 3.66(3 \mathrm{H}, \mathrm{s}) 3.60(1 \mathrm{H}, \mathrm{m})$ $2.33(1 \mathrm{H}, \mathrm{dd}, J=15.7,6.2 \mathrm{~Hz}) 2.18(1 \mathrm{H}, \mathrm{dd}, J=15.7,2.4 \mathrm{~Hz}) 1.61(3 \mathrm{H}, \mathrm{d}, J=6.2 \mathrm{~Hz}) 1.49$ $(3 \mathrm{H}, \mathrm{d}, J=7.2 \mathrm{~Hz}) ;{ }^{13} \mathrm{C}$ NMR $\left(500 \mathrm{MHz}, \mathrm{CDCl}_{3}\right) \delta 188.1,170.8,170.7,144.1,132.7,129.4$, 128.2, 128.2, 127.3, 96.4, 51.8, 51.4, 50.4, 43.4, 17.8, 17.3. HRMS $\mathrm{C}_{18} \mathrm{H}_{21} \mathrm{NO}_{4} \mathrm{Na}$ calcd. 338.1368 , found 338.1360 .

(1S,6S)-1-( $\alpha$-Methylbenzyl)-6-methylpiperidine-2,4-dione (12a). Following general procedure $\mathrm{C}$, reaction of diester 10a $(0.23 \mathrm{~g}, 0.70 \mathrm{mmol})$, and sodium methoxide in methanol (2.0 M, $0.70 \mathrm{~mL}, 1.4 \mathrm{mmol})$ in methanol $(5 \mathrm{~mL})$ gave $12 \mathrm{a}(0.16 \mathrm{~g}, 97 \%)$ as a pale yellow oil, $[\alpha]_{\mathrm{D}}{ }^{21}=-198.1\left(c 3.09, \mathrm{CHCl}_{3}\right)$; IR $v_{\max } 2975,1730,1642 \mathrm{~cm}^{-1} ;{ }^{1} \mathrm{H} \mathrm{NMR}\left(500 \mathrm{MHz}, \mathrm{CDCl}_{3}\right) \delta$ 7.36-7.25 (5H, m) $6.10(1 \mathrm{H}, \mathrm{q}, J=6.9 \mathrm{~Hz}) 3.55(1 \mathrm{H}, \mathrm{m}) 3.41-3.29(2 \mathrm{H}, \mathrm{m}) 2.24(1 \mathrm{H}, \mathrm{dd}, J=$ 16.5, 8.6 Hz) $2.17(1 \mathrm{H}, \mathrm{dd}, J=16.5,5.0 \mathrm{~Hz}) 1.59(3 \mathrm{H}, \mathrm{d}, J=6.9 \mathrm{~Hz}) 1.25(3 \mathrm{H}, \mathrm{d}, J=6.7 \mathrm{~Hz})$; ${ }^{13} \mathrm{C}$ NMR $\left(500 \mathrm{MHz}, \mathrm{CDCl}_{3}\right) \delta$ 204.6, 166.2, 140.0, 128.8, 128.1, 127.2, 50.9, 47.4, 46.5, 45.1, 22.7, 16.9; m/z (CI, \%) $232\left([\mathrm{M}+\mathrm{H}]^{+}, 100\right) 105\left(\mathrm{C}_{8} \mathrm{H}_{9}{ }^{+}, 35\right)$. HRMS $\mathrm{C}_{14} \mathrm{H}_{18} \mathrm{NO}_{2}$ calcd. 232.1338 , found 232.1334 .

(1S,6R)-1-( $\alpha$-Methylbenzyl)-6-phenylpiperidine-2,4-dione (12f). The piperidine-2,4-dione sodium salt $11 \mathrm{f}(0.10 \mathrm{~g}, 0.28 \mathrm{mmol})$ was added to hydrochloric acid $(1.3 \mathrm{M}, 5 \mathrm{~mL})$. The mixture was heated under reflux for $1 \mathrm{~h}$, then allowed to cool to $20{ }^{\circ} \mathrm{C}$ and extracted with dichloromethane $(2 \times 5 \mathrm{~mL})$. The combined organic layers were dried $\left(\mathrm{MgSO}_{4}\right)$ and evaporated, and the residue was purified by flash chromatography (1:1 ethyl acetate:hexane) to give $12 \mathrm{f}(69 \mathrm{mg}, 85 \%)$ as pale yellow solid, $\mathrm{mp} 104-107{ }^{\circ} \mathrm{C} ;[\alpha]_{\mathrm{D}}{ }^{21}=+103.4(c 2.95$, $\mathrm{CHCl}_{3}$ ); IR $v_{\max } 2923,1730,1648 \mathrm{~cm}^{-1} ;{ }^{1} \mathrm{H} \mathrm{NMR}\left(500 \mathrm{MHz}, \mathrm{CDCl}_{3}\right) \delta 7.39-7.11(10 \mathrm{H}, \mathrm{m})$ $6.31(1 \mathrm{H}, \mathrm{q}, J=7.2 \mathrm{~Hz}) 4.65(1 \mathrm{H}, \mathrm{dd}, J=6.1,2.2 \mathrm{~Hz}) 3.39(2 \mathrm{H}, \mathrm{s}) 2.68(1 \mathrm{H}, \mathrm{dd}, J=16.4,2.2$ Hz) $2.51(1 \mathrm{H}, \mathrm{dd}, J=16.4,6.1 \mathrm{~Hz}) 1.35(3 \mathrm{H}, \mathrm{d}, J=7.2 \mathrm{~Hz}) ;{ }^{13} \mathrm{C} \mathrm{NMR}\left(500 \mathrm{MHz}, \mathrm{CDCl}_{3}\right) \delta$ 203.2, 167.4, 140.1, 140.0, 129.2, 128.9, 128.1, 128.0, 127.5, 125.7, 53.0, 51.6, 48.8, 47.9, 16.4; $m / z(\mathrm{EI}, \%) 293\left([\mathrm{M}+\mathrm{H}]^{+}, 100\right) 105\left(\mathrm{C}_{8} \mathrm{H}_{9}{ }^{+}, 35\right) 77\left(\mathrm{C}_{6} \mathrm{H}_{5}{ }^{+}, 10\right)$. HRMS $\mathrm{C}_{19} \mathrm{H}_{19} \mathrm{NO}_{2}$ calcd. 293.1416, found 293.1413.

(1S,6R)-1-( $\alpha$-Methylbenzyl)-6-(pyridin-3-yl)piperidine-2,4-dione (12g). Following general procedure $\mathrm{C}$, reaction of diester $10 \mathrm{~g}(0.25 \mathrm{~g}, 0.65 \mathrm{mmol})$, and sodium methoxide in methanol $(2.0 \mathrm{M}, 0.65 \mathrm{~mL}, 1.30 \mathrm{mmol})$ in methanol $(3 \mathrm{~mL})$ gave $\mathbf{1 2 \mathrm { g }}(0.19 \mathrm{~g}, 99 \%)$ as a brown oil, $[\alpha]_{\mathrm{D}}^{25}=-84.7\left(c 1.00, \mathrm{CHCl}_{3}\right) ; \mathrm{IR} v_{\max } 2977,1730,1642 \mathrm{~cm}^{-1} ;{ }^{1} \mathrm{H} \mathrm{NMR}\left(500 \mathrm{MHz}, \mathrm{CDCl}_{3}\right) \delta$ $8.57(1 \mathrm{H}, \mathrm{d}, J=4.5 \mathrm{~Hz}) 8.48(1 \mathrm{H}, \mathrm{s}) 7.65(1 \mathrm{H}, \mathrm{m}) 7.53-7.26(6 \mathrm{H}, \mathrm{m}) 6.31(1 \mathrm{H}, \mathrm{q}, J=7.2 \mathrm{~Hz})$ $4.66(1 \mathrm{H}, \mathrm{dd}, J=6.0,1.8 \mathrm{~Hz}) 3.46(1 \mathrm{H}, \mathrm{d}, J=20.6 \mathrm{~Hz}) 3.35(1 \mathrm{H}, \mathrm{d}, J=20.6 \mathrm{~Hz}) 2.66(1 \mathrm{H}, \mathrm{dd}$, $J=16.4,1.8 \mathrm{~Hz}) 2.56(1 \mathrm{H}, \mathrm{dd}, J=16.4,6.0 \mathrm{~Hz}) 1.34(3 \mathrm{H}, \mathrm{d}, J=7.2 \mathrm{~Hz}) ;{ }^{13} \mathrm{C} \mathrm{NMR}(500 \mathrm{MHz}$, $\left.\mathrm{CDCl}_{3}\right) \delta 202.1,167.1,149.6,147.6,139.5,135.7,133.2,132.2,129.1,128.6,128.3,127.4$, 
123.8, 51.6, 51.1, 48.6, 47.7, 16.6; m/z (EI, \%) $294\left(\mathrm{M}^{+}, 15\right)$. HRMS $\mathrm{C}_{18} \mathrm{H}_{18} \mathrm{~N}_{2} \mathrm{O}_{2}$ calcd. 294.1368, found 294.1366.

(1S,6R)-1-( $\alpha$-Methylbenzyl)-6-isopropylpiperidine-2,4-dione (12j). Following general procedure $\mathrm{C}$, reaction of diester $\mathbf{1 0 j}(0.15 \mathrm{~g}, 0.47 \mathrm{mmol})$ and sodium methoxide in methanol $(2.0 \mathrm{M}, 0.50 \mathrm{~mL}, 1.0 \mathrm{mmol})$ in methanol ( $3 \mathrm{~mL})$ afforded $\mathbf{1 2} \mathbf{j}(0.12 \mathrm{~g}, 95 \%)$ as a pale yellow oil, $[\alpha]_{\mathrm{D}}{ }^{21}=-177.9\left(c 1.31, \mathrm{CHCl}_{3}\right)$; IR $v_{\max } 2968,1726,1644 \mathrm{~cm}^{-1} ;{ }^{1} \mathrm{H}$ NMR $(500 \mathrm{MHz}$, $\left.\mathrm{CDCl}_{3}\right) \delta 7.37-7.26(5 \mathrm{H}, \mathrm{m}) 6.07(1 \mathrm{H}, \mathrm{q}, J=7.1 \mathrm{~Hz}) 3.42(1 \mathrm{H}, \mathrm{d}, J=21.3 \mathrm{~Hz}) 3.20(1 \mathrm{H}, \mathrm{d}, J=$ $21.3 \mathrm{~Hz}) 3.16(1 \mathrm{H}, \mathrm{m}) 2.52(1 \mathrm{H}, \mathrm{dd}, J=16.7,5.0 \mathrm{~Hz}) 2.02-1.96(2 \mathrm{H}, \mathrm{m}) 1.65(3 \mathrm{H}, \mathrm{d}, J=7.1$ Hz) $0.90(3 \mathrm{H}, \mathrm{d}, J=6.9 \mathrm{~Hz}) 0.84(3 \mathrm{H}, \mathrm{d}, J=6.9 \mathrm{~Hz}) ;{ }^{13} \mathrm{C} \mathrm{NMR}\left(500 \mathrm{MHz}, \mathrm{CDCl}_{3}\right) \delta 208.2$, $170.2,142.5,131.5,130.7,130.2,58.3,55.4,50.1,43.7,35.9,22.9,20.4,20.2 ; \mathrm{m} / \mathrm{z}\left(\mathrm{EI}^{+}\right) 259$ $\left(\mathrm{M}^{+}, 100\right) 216\left(\left[\mathrm{M}-\mathrm{C}_{3} \mathrm{H}_{7}\right]^{+}, 99\right) 105\left(\mathrm{C}_{8} \mathrm{H}_{9}^{+}, 75\right) 77\left(\mathrm{C}_{6} \mathrm{H}_{5}^{+}, 40\right)$. HRMS $\mathrm{C}_{16} \mathrm{H}_{21} \mathrm{NO}_{2}$ calcd. 259.1572 , found 259.1575 .

(1S,6R)-1-( $\alpha$-Methylbenzyl)-6-cyclohexylpiperidine-2,4-dione (12k). Following general procedure $\mathrm{C}$, reaction of diester $10 \mathrm{k}(0.22 \mathrm{~g}, 0.57 \mathrm{mmol})$ and sodium methoxide in methanol $(2.0 \mathrm{M}, 0.6 \mathrm{~mL}, 1.2 \mathrm{mmol})$ in methanol $(3 \mathrm{~mL})$ gave $\mathbf{1 2 k}(0.13 \mathrm{~g}, 76 \%)$ as a colourless oil, $[\alpha]_{\mathrm{D}}{ }^{25}=-133.1\left(c 3.40, \mathrm{CHCl}_{3}\right)$; IR $v_{\max } 2926,1726,1640 \mathrm{~cm}^{-1} ;{ }^{1} \mathrm{H} \mathrm{NMR}\left(500 \mathrm{MHz}, \mathrm{CDCl}_{3}\right) \delta$ 7.46-7.25 (5H, m) $6.03(1 \mathrm{H}, \mathrm{q}, J=7.2 \mathrm{~Hz}) 3.38(1 \mathrm{H}, \mathrm{d}, J=21.2 \mathrm{~Hz}) 3.26(1 \mathrm{H}, \mathrm{d}, J=21.2 \mathrm{~Hz})$ $3.13(1 \mathrm{H}, \mathrm{m}) 2.49(1 \mathrm{H}, \mathrm{dd}, J=16.7,3.6 \mathrm{~Hz}) 1.95(1 \mathrm{H}, \mathrm{dd}, J=16.7,6.5 \mathrm{~Hz}) 1.74(2 \mathrm{H}, \mathrm{m}) 1.61$ $(3 \mathrm{H}, \mathrm{d}, J=7.2 \mathrm{~Hz}) 1.59-1.47(3 \mathrm{H}, \mathrm{m}) 1.14-0.80(6 \mathrm{H}, \mathrm{m}) ;{ }^{13} \mathrm{C} \mathrm{NMR}\left(500 \mathrm{MHz}, \mathrm{CDCl}_{3}\right) \delta$ 205.6, 169.5, 139.9, 128.8, 128.0, 127.5, 55.2, 52.8, 47.7, 43.6, 41.7, 30.7, 28.6, 26.6, 26.4, 26.0, $17.6 ; \mathrm{m} / \mathrm{z}(\mathrm{CI}, \%) 300\left([\mathrm{M}+\mathrm{H}]^{+}, 100\right)$. HRMS $\mathrm{C}_{19} \mathrm{H}_{26} \mathrm{NO}_{2}$ calcd. 300.1964, found 300.1953 .

(1S,6R)-1-( $\alpha$-Methylbenzyl)-6-(E)-propenylpiperidine-2,4-dione (12l). The piperidine-2,4dione sodium salt 111 (1.0 g, $2.96 \mathrm{mmol})$ was added to hydrochloric acid $(1.3 \mathrm{M}, 30 \mathrm{~mL})$. The mixture was heated under reflux for $1 \mathrm{~h}$, then allowed to cool to $20{ }^{\circ} \mathrm{C}$ and extracted with dichloromethane $(2 \times 30 \mathrm{~mL})$. The combined organic layers were dried $\left(\mathrm{MgSO}_{4}\right)$ and evaporated to give $12 \mathrm{l}(0.56 \mathrm{~g}, 74 \%)$ as a pale yellow oil, $[\alpha]_{\mathrm{D}}{ }^{21}=-203.8\left(c 2.08, \mathrm{CHCl}_{3}\right)$; IR $v_{\max } 3060,2938,1726,1650 \mathrm{~cm}^{-1} ;{ }^{1} \mathrm{H}$ NMR $\left(300 \mathrm{MHz}, \mathrm{CDCl}_{3}\right) \delta 7.52-7.26(5 \mathrm{H}, \mathrm{m}) 6.14(1 \mathrm{H}$, q, $J=7.2 \mathrm{~Hz}) 5.55(1 \mathrm{H}, \mathrm{ddq}, J=18.0,6.2,1.4 \mathrm{~Hz}) 5.41(1 \mathrm{H}, \mathrm{dq}, J=18.0,1.7 \mathrm{~Hz}) 3.92(1 \mathrm{H}, \mathrm{m})$ 3.41-3.24 (2H, m) $2.42(1 \mathrm{H}, \mathrm{dd}, J=17.0,2.1 \mathrm{~Hz}) 2.16(1 \mathrm{H}, \mathrm{dd}, J=17.0,5.1 \mathrm{~Hz}) 1.68(3 \mathrm{H}, \mathrm{dd}$, $J=6.2,1.7 \mathrm{~Hz}) 1.54(3 \mathrm{H}, \mathrm{d}, J=7.2 \mathrm{~Hz}) ;{ }^{13} \mathrm{C} \mathrm{NMR}\left(300 \mathrm{MHz}, \mathrm{CDCl}_{3}\right) \delta 204.1,166.6,139.9$, $129.8,128.7,128.0,127.9,127.3,51.1,50.2,48.4,44.9,17.7,16.5 ; \mathrm{m} / \mathrm{z}\left(\mathrm{CI}^{+}\right) 257\left(\mathrm{M}^{+}, 70\right)$ $105\left(\mathrm{C}_{8} \mathrm{H}_{9}{ }^{+}, 39\right) 77\left(\mathrm{C}_{6} \mathrm{H}_{5}{ }^{+}, 15\right)$. HRMS $\mathrm{C}_{16} \mathrm{H}_{19} \mathrm{NO}_{2}$ calcd. 257.1416, found 257.1428. 
(6R)-Phenylpiperidine-2,4-dione ( $\boldsymbol{R})$-(6f). Methanesulfonic acid (0.23 $\mathrm{mL}, 3.07 \mathrm{mmol})$ was added to a stirred solution of piperidine-2,4-dione $\mathbf{1 2 f}(1.0 \mathrm{~g}, 3.41 \mathrm{mmol})$ in toluene $(15 \mathrm{~mL})$ at $20^{\circ} \mathrm{C}$, under nitrogen. The mixture was then heated under reflux for $3 \mathrm{~h}$, allowed to cool to $20{ }^{\circ} \mathrm{C}$, evaporated, and the residue was purified by flash chromatography (9:1 ethyl acetate:hexane) to give $(\boldsymbol{R})-\mathbf{6 f}(0.36 \mathrm{~g}, 56 \%)$ as a white solid, mp $163-166{ }^{\circ} \mathrm{C}$ (lit. ${ }^{38} 166-168$ $\left.{ }^{\circ} \mathrm{C}\right) ;[\alpha]_{\mathrm{D}}{ }^{25}=+119.1\left(c 1.00, \mathrm{CHCl}_{3}\right)\left(\mathrm{lit}^{38}{ }^{38}[\alpha]_{\mathrm{D}}{ }^{20}=+124.3 c 0.35, \mathrm{CHCl}_{3}\right)$; IR $v_{\max } 3185,2899$, 1716, $1665 \mathrm{~cm}^{-1} ;{ }^{1} \mathrm{H}$ NMR $\left(500 \mathrm{MHz}, \mathrm{CDCl}_{3}\right) \delta$ 7.43-7.21 $(5 \mathrm{H}, \mathrm{m}) 7.13(1 \mathrm{H}$, br s) $4.89(1 \mathrm{H}$, m) $3.33(2 \mathrm{H}, \mathrm{s}) 2.85(1 \mathrm{H}, \mathrm{dd}, J=16.1,4.5 \mathrm{~Hz}) 2.73(1 \mathrm{H}, \mathrm{dd}, J=16.1,8.9 \mathrm{~Hz}) ;{ }^{13} \mathrm{C}$ NMR $(500$ $\left.\mathrm{MHz}, \mathrm{CDCl}_{3}\right) \delta 202.5,169.2,139.4,129.4,128.9,126.1,52.9,47.3,47.0 ; \mathrm{m} / \mathrm{z}$ (EI, \%) 189 $\left(\mathrm{M}^{+}, 100\right)$. HRMS $\mathrm{C}_{11} \mathrm{H}_{11} \mathrm{NO}_{2}$ calcd. 189.0790, found 189.0787.

(6R)-Isopropylpiperidine-2,4-dione $(\boldsymbol{R})$-(6j). Methanesulfonic acid $(0.02 \mathrm{~mL}, 0.26 \mathrm{mmol})$ was added to a stirred solution of piperidine-2,4-dione $\mathbf{1 2} \mathbf{j}$ ( $76 \mathrm{mg}, 0.29 \mathrm{mmol}$ ) in toluene (2 $\mathrm{mL}$ ) at $20^{\circ} \mathrm{C}$, under nitrogen. The mixture was heated under reflux for $3 \mathrm{~h}$, then allowed to cool, evaporated, and the residue was purified by flash chromatography (ethyl acetate) to give $(\boldsymbol{R})-\mathbf{6 j}(22 \mathrm{mg}, 48 \%)$ as a pale yellow oil; $[\alpha]_{\mathrm{D}}{ }^{21}=+30.6\left(c 0.49, \mathrm{CHCl}_{3}\right), \mathrm{lit}^{37}[\alpha]_{\mathrm{D}}{ }^{13}=+35.3$ (c 1.00, MeOH); IR $v_{\max } 3212,2922,1722,1665 \mathrm{~cm}^{-1} ;{ }^{1} \mathrm{H}$ NMR $\left(500 \mathrm{MHz}, \mathrm{CDCl}_{3}\right) \delta 7.05$ $(1 \mathrm{H}, \mathrm{br} \mathrm{s}) 3.49(1 \mathrm{H}, \mathrm{m}) 3.28(2 \mathrm{H}, \mathrm{s}) 2.63(1 \mathrm{H}, \mathrm{dd}, J=16.1,4.4 \mathrm{~Hz}) 2.45(1 \mathrm{H}, \mathrm{dd}, J=16.1,9.1$ Hz) $1.82(1 \mathrm{H}, \mathrm{m}) 1.01-0.95(6 \mathrm{H}, \mathrm{m}) ;{ }^{13} \mathrm{C} \mathrm{NMR}\left(500 \mathrm{MHz}, \mathrm{CDCl}_{3}\right) \delta 203.5,170.2,54.3,47.2$, 41.6, 32.5, 18.2, 17.9; $\mathrm{m} / \mathrm{z}$ (ES, \%) $155\left(\mathrm{M}^{+}, 50\right)$. HRMS $\mathrm{C}_{8} \mathrm{H}_{13} \mathrm{NO}_{2}$ calcd. 155.0946, found 155.0940 .

(6R)-Cyclohexylpiperidine-2,4-dione (R)-(6k). Methanesulfonic acid (0.04 mL, $0.61 \mathrm{mmol})$ was added to a stirred solution of piperidine-2,4-dione $12 \mathbf{k}(0.14 \mathrm{~g}, 0.47 \mathrm{mmol})$ in toluene (3 $\mathrm{mL}$ ) at $20^{\circ} \mathrm{C}$, under nitrogen. The mixture was heated under reflux for $5 \mathrm{~h}$, then allowed to cool to $20^{\circ} \mathrm{C}$, and evaporated to give an oil that was purified by flash chromatography (ethyl acetate) to give $(\boldsymbol{R})-\mathbf{6 k}(38 \mathrm{mg}, 42 \%)$ as yellow solid, $\mathrm{mp} 125-127^{\circ} \mathrm{C} ;[\alpha]_{\mathrm{D}}{ }^{21}=+14.2(\mathrm{c} 1.26$, $\mathrm{MeOH}) ; \mathrm{IR} v_{\max } 3223,2925,1724,1665 \mathrm{~cm}^{-1} ;{ }^{1} \mathrm{H}$ NMR (500 MHz, $\left.\mathrm{CDCl}_{3}\right) \delta 7.10(1 \mathrm{H}, \mathrm{br} \mathrm{s})$ $3.55(1 \mathrm{H}, \mathrm{m}) 3.25(2 \mathrm{H}, \mathrm{s}) 2.61(1 \mathrm{H}, \mathrm{dd}, J=16.1,4.8 \mathrm{~Hz}) 2.50(1 \mathrm{H}, \mathrm{dd}, J=16.1,8.1 \mathrm{~Hz}) 1.80$ $1.74(3 \mathrm{H}, \mathrm{m}) 1.70-1.69(2 \mathrm{H}, \mathrm{m}) 1.60(1 \mathrm{H}, \mathrm{m}) 1.25-1.14(3 \mathrm{H}, \mathrm{m}) 1.00(2 \mathrm{H}, \mathrm{m}) ;{ }^{13} \mathrm{C}$ NMR $(500$ $\left.\mathrm{MHz}, \mathrm{CDCl}_{3}\right) \delta$ 203.9, 169.4, 53.6, 47.3, 42.4, 41.7, 28.8, 28.5, 26.1, 25.9 25.8; $\mathrm{m} / \mathrm{z}(\mathrm{EI}, \%)$ $195\left(\mathrm{M}^{+}, 2\right) 112\left(\left[\mathrm{M}-\mathrm{C}_{6} \mathrm{H}_{11}\right]^{+}, 100\right)$. HRMS C $\mathrm{H}_{11} \mathrm{H}_{17} \mathrm{NO}_{2}$ calcd. 195.1259, found 195.1255.

(1S,6R)-1-( $\alpha$-Methylbenzyl)-3,3-dimethyl-6-(E)-propenylpiperidine-2,4-dione $\quad(13) . \quad$ To piperidine-2,4-dione $12 \mathrm{l}(0.13 \mathrm{~g}, 0.51 \mathrm{mmol})$ in ethanol $(2 \mathrm{~mL})$ was added potassium carbonate $(0.21 \mathrm{~g}, 1.52 \mathrm{mmol})$ and methyl iodide $(0.1 \mathrm{~mL}, 1.52 \mathrm{mmol})$. The mixture was 
stirred at $40{ }^{\circ} \mathrm{C}$ for $16 \mathrm{~h}$ then filtered. The filtrate was evaporated and the residue was dissolved in chloroform $(5 \mathrm{~mL})$ and the solution was filtered. Evaporation afforded an oil that was purified by flash chromatography (15:85 ethyl acetate:hexane) to give $\mathbf{1 3}$ (0.11 g, 76\%) as a colourless oil, $[\alpha]_{\mathrm{D}}{ }^{21}=-142.9\left(c 0.35, \mathrm{CHCl}_{3}\right)$; IR $v_{\max } 2923,1724,1640 \mathrm{~cm}^{-1} ;{ }^{1} \mathrm{H}$ NMR $\left(300 \mathrm{MHz}, \mathrm{CDCl}_{3}\right) \delta$ 7.36-7.24 $(5 \mathrm{H}, \mathrm{m}) 6.07(1 \mathrm{H}, \mathrm{q}, J=6.5 \mathrm{~Hz}) 5.48(1 \mathrm{H}, \mathrm{m}) 5.25(1 \mathrm{H}, \mathrm{m})$ $3.82(1 \mathrm{H}, \mathrm{m}) 2.65(1 \mathrm{H}, \mathrm{dd}, J=13.8,5.7 \mathrm{~Hz}) 2.30(1 \mathrm{H}, \mathrm{dd}, J=13.8,2.5 \mathrm{~Hz}) 1.63(3 \mathrm{H}, \mathrm{d}, J=6.5$ Hz) $1.51(3 \mathrm{H}, \mathrm{dd}, J=7.2,1.8 \mathrm{~Hz}) 1.38(3 \mathrm{H}, \mathrm{d}, J=1.9 \mathrm{~Hz}) 1.35(3 \mathrm{H}, \mathrm{d}, J=1.9 \mathrm{~Hz}) ;{ }^{13} \mathrm{C}$ NMR (300 MHz, $\left.\mathrm{CDCl}_{3}\right) \delta$ 209.2, 174.0, 140.5, 131.1, 128.7, 128.3, 127.7, 127.3, 52.4, 52.1, 50.6, 44.4, 26.5, 21.7, 17.6, 16.8; m/z (EI, \%) $286\left(\mathrm{M}^{+}, 4\right) ; 105\left(\mathrm{C}_{8} \mathrm{H}_{9}{ }^{+}, 100\right)$. HRMS $\mathrm{C}_{18} \mathrm{H}_{24} \mathrm{NO}_{2}$ calcd. 286.1807, found 286.1803 .

(4R,6R)-4-Hydroxy-6-phenylpiperidin-2-one (14). Zinc borohydride (2.0 mL, 1.37 M, 2.74 mmol) was added to a stirred solution of the piperidine-2,4-dione $(\boldsymbol{R})-\mathbf{6 f}(0.19 \mathrm{~g}, 0.98 \mathrm{mmol})$ in anhydrous dichloromethane $(2 \mathrm{~mL})$ at $0{ }^{\circ} \mathrm{C}$, under nitrogen. The mixture was then stirred at $20{ }^{\circ} \mathrm{C}$ for $20 \mathrm{~h}$. Water $(5 \mathrm{~mL})$ was added to the mixture which was then extracted with ethyl acetate $(2 \times 10 \mathrm{~mL})$. The combined organic layers were dried $\left(\mathrm{MgSO}_{4}\right)$ and evaporated. The residue was recrystallised from isopropanol-hexane to give $\mathbf{1 4}(0.08 \mathrm{~g}, 43 \%)$ as a white solid, mp 206-210 ${ }^{\circ} \mathrm{C}\left(\right.$ lit. $\left.^{10 \mathrm{~b}} 213{ }^{\circ} \mathrm{C}\right) ;[\alpha]_{\mathrm{D}}{ }^{25}=+53.3(c 1.20, \mathrm{MeOH})$, lit. $^{10 \mathrm{~b}}[\alpha]_{\mathrm{D}}{ }^{20}=+52.3(c 0.88$, $\mathrm{MeOH}$ ); IR $v_{\max } 3269,2896,1648 \mathrm{~cm}^{-1} ;{ }^{1} \mathrm{H}$ NMR (500 MHz, $\left.\mathrm{CD}_{3} \mathrm{OD}\right) \delta$ 7.38-7.28 (5H, m) $4.51(1 \mathrm{H}, \mathrm{dd}, J=11.6,4.3 \mathrm{~Hz}) 4.11(1 \mathrm{H}, \mathrm{m}) 2.71(1 \mathrm{H}, \mathrm{ddd}, J=17.1,5.6,2.3 \mathrm{~Hz}) 2.33-2.26$ $(2 \mathrm{H}, \mathrm{m}) 1.62(1 \mathrm{H}, \mathrm{ddd}, J=15.6,11.6,10.8 \mathrm{~Hz}) ;{ }^{13} \mathrm{C} \mathrm{NMR}\left(500 \mathrm{MHz}, \mathrm{CD}_{3} \mathrm{OD}\right) \delta 174.1,143.5$, 129.8, 129.0, 127.4, 65.6, 56.2, 42.7, 41.4; m/z (CI, \%) $192\left(\mathrm{M}^{+}, 100\right)$. HRMS C $\mathrm{C}_{11} \mathrm{H}_{14} \mathrm{NO}_{2}$ calcd. 192.1025, found 192.1028 .

\section{Charles M. Marson* and Kin Cheung Yau}

Department of Chemistry, University College London, Christopher Ingold Laboratories, 20

Gordon Street, London WC1H OAJ, U.K.E-mail: c.m.marson@ucl.ac.uk;

Fax: +(44) (0)207679 7463; tel: +(44) (0)2076794712.

\section{References}

1. Vitaku, E.; Smith, D. T.; Njardarson, T. J. Med. Chem. 2014, 57, 10257.

2. (a) Michael, J. P. Nat. Prod. Rep. 1999, 16, 675. (b) Daly, J. W. J. Nat. Prod. 1998, 61, 162. (c) Schneider, M. Pyridine and piperidine alkaloids: an update, in: Alkaloids: chemical and biochemical perspectives, vol. 10 (Ed.: S. W. Pelletier), Elsevier Science, Oxford, UK, 1996, 155. (d) Strunz, G. M.; Findlay, J. A. Pyridine and Piperidine Alkaloids, in: The Alkaloids (Ed.: A. Brossi), Academic Press, New York, 1985, vol. 26, pp. 89-183. (e) Fodor, G. B.; Colasanti, B. in Alkaloids: Chemical and Biological Perspectives (Ed.: S. W. Pelletier), Wiley, New York, 1985, vol. 3, pp. 1-90; (f) Jones, T. H.; Blum, M. S. in Alkaloids: Chemical and Biological Perspectives, ed. S. W. Pelletier, Wiley, New York, 1983, vol. 1, pp. 33-84.

3. (a) Jones, T. H.; Blum, M. S. in Alkaloids: Chemical and Biological Perspectives, ed. S. W. Pelletier, Wiley, New York, 1985, vol. 3, pp. 1-90 (b) Lognay, G.; Hemptinne, J. L.; Chan, 
F. Y.; Gaspar, CH.; Marlier, M. N.; Braekman, J. C.; Daloze, D.; Pasteels, J. M. J. Nat. Prod. 1996, 59, 510. (c) Williams, P. G.; Yoshida, W. Y.; Moore, R. E.; Paul, V. J. J. Nat. Prod. 1996, 66, 620. (d) Bonjoch, J.; Solé, D. Chem. Rev. 2000, 100, 3455.

4. (a) Pei, Z.; Li, X.; von Geldren, T. W.; Longenecker, K.; Pieh, D.; Stewart, K. D.; Backes, B. J.; Lai, C.; Lubben, T. H.; Balleron, S. J.; Beno, D. W. A.; Kempf-Grote, A. J.; Sham, H. L.;

Trevillyan, J. M. J. Med. Chem. 2005, 50, 1983. (b) Reichard, G. A.; Spitler, J.; Mergelsberg, I.; Miller, A.; Wong, G.; Raghavan, R.; Jenkins, J.; Gan, T.; McPhail, A. T. Tetrahedron: Asymmetry 2002, 13, 939. (c) Nishimura, Y.; Adachi, H.; Satoh, T.; Shitara, E.; Nakamura, H.; Kojima, F.; Takeuchi, T. J. Org. Chem. 2000, 65, 4871.

5. (a) Buffat, M. G. P. Tetrahedron 2004, 60, 1701. (b) Weintraub, P. M.; Sabol, J. S.; Kane, J. M.; Borcherding, D. R. Tetrahedron 2003, 59, 2953.

6. (a) Xin, D.; Perez, L. M.; Ioerger, T. R.; Burgess, K. Angew. Chem. Int. Ed. 2014, 53, 3594. (b) Menichincheri, M.; Bargiotti, A.; Berthelsen, J.; Bertrand, J. A.; Bossi, R.; Ciavolella, A.; Cirla, A.; Cristiani, C.; Croci, V.; D’Alessio, R.; Fasolini, M.; Fiorentini, F.; Forte, B.; Isacchi, A.; Martina, K.; Molinari, A.; Montagnoli, A.; Orsini, P.; Orzi, F.; Pesenti, E.; Pezzetta, D.; Pillan, A.; Poggesi, I.; Roletto, F.; Scolaro, A.; Tato, M.; Tibolla, M.; Valsasina, B.; Varasi, M.; Volpi, D.; Santocanale, C.; Vanotti, E. J. Med. Chem. 2009, 52, 293. (c) Xie, Y.; Raffo, A.; Ichise, M.; Deng, S.; Harris, P. E.; Landry, D. W. Bioorg. Med. Chem. Lett. 2008, 18, 5111. (d) Leflemme, N.; Dallemagne, P.; Rault, S. Tetrahedron Lett. 2001, 42, 8997. (e) Desmaële, D.; Mekouar, K.; d'Angelo, J. J. Org. Chem. 1997, 62, 3890. (f) Vanotti, E.; Menichincheri, M.; Orsini, P.; Scolaro, A.; Varasi, M. US2007/142414 Pharmacia Italia, 21.06.07. (g) Macdonald, G. J.; Trabanco-Suarez, A. A.; Conde-Seide, S.; Tresadern, G. J.; Bartolome-Nebreda, J. M.; PastorFernandez, J. WO2011/073339 Janssen Pharmaceutica, 23.06.11.

7. (a) Thaker, M. N.; García, M.; Koteva, K.; Waglechner, N.; Sorensen, D.; Medina, R.; Wright, G. D. Med. Chem. Commun. 2012, 3, 1020. (b) Rowlands, M. G.; Bunnett, M. A.; Foster, A. B.; Jarman, M.; Stanek, J.; Schweizer, E. J. Med. Chem. 1988, 31, 971. (c) Leonardi, A.; Barlocco, D.; Montesano, F.; Cignarella, G.; Motta, G.; Testa, R.; Poggesi, E.; Seeber, M.; De Benedetti, P. G.; Fanelli, F. J. Med. Chem. 2004, 47, 1900.

8. Kuznetsov, N. Y.; Maleev, V. I.; Khrustalev, V. N.; Mkrtchyan, A. F.; Godovikov, I. A.; Strelkova, T. V.; Bubnov, Y. N. Eur. J. Org. Chem. 2012, 2, 334.

9. Comins, D. L.; Zhang, Y.; Joseph, S. P. Org. Lett. 1999, 1, 657.

10. (a) Davis, F. A.; Chao, B.; Rao, A. Org. Lett. 2001, 3, 3169. (b) Davis, F. A.; Fang, T.; Chao, B.; Burns, D. M. Synthesis, 2000, 14, 2106.

11. Li, X.; Taechalertpaisarn, J.; Xin, D.; Burgess, K. Org. Lett. 2015, 17, 632.

12. Huckin, S. N.; Weiler, L. J. Am. Chem. Soc. 1974, 96, 1082.

13. Ashton, M. J.; Hills, S. J.; Newton, C. G.; Taylor, J. B.; Tondu, S. C. D. Heterocycles 1989, 28, 1015-1035.

14. Prévost, N.; Shipman, M. Tetrahedron 2002, 58, 7165.

15. Grauert, M.; Bechtel, W. D.; Weiser, T.; Stransky, W.; Nar, H.; Carter, A. J. J. Med. Chem. 2002, 45, 3755 .

16. Bulman Page, P. C.; Hamzah, A. S.; Leach, D. C.; Allin, S. M.; Andrews, D. M.; Rassias, G. A. Org. Lett. 2003, 5, 353.

17. Krapcho, P. A.; Weimaster, J. F.; Eldridge, J. M.; Jahngen, E. G. E.; Lovey, A. J.; Stephens, W. P. Org. Lett. 1978, 43, 138.

18. (a) Davies, S. G.; Fletcher, A. M.; Roberts, P. M.; Smith, A. D. Tetrahedron 2009, 65, 10192.

(b) Davies, S. G.; Garner, A. C.; Nicholson, R. I.; Osborne, J.; Roberts, P. M.; Savory, E. D.;

Smith, A. D.; Thomson, J. E. Org. Biomol. Chem. 2009, 12, 2604.

19. Laguzza, B. C.; Ganem, B. Tetrahedron Lett. 1978, 19, 1689.

20. Zacuto, M. J.; Xu, F. J. Org. Chem. 2007, 72, 6298.

21. Paik, S.; Lee, J. Y. Tetrahedron Lett. 2006, 47, 1813.

22. Davis, F. A.; Chao, B.; Andemichael, Y. W.; Mohanty, P. K.; Fang, T.; Burns, D. M.; Rao, A.; Szewczyk, J. M. Heteratom Chem. 2002, 13, 486.

23. Rioz-Martinez, A.; Shaik, A. A.; Azeeza, S.; Malik, M. S.; Sandbhor, M. Angew. Chem. Int. Ed. 2011, 50, 8387 .

24. Adriaensens, L. V.; Hartley, R. C. J. Org. Chem. 2007, 72, 10287.

25. Weis, R.; Presser, A.; Seebacher, W. Monatsch. Chem. 2003, 134, 1129.

26. Cardillo, G.; Galeazzi, R.; Mobbili, G.; Orena, M.; Rossetti, M. Heterocycles 1994, 2663.

27. Cardillo, G.; Gentilucci, L.; Tolomelli, A.; Tomasini, C. J. Org. Chem. 1998, 63, 2351.

28. Joel, S. P.; Marson, C. M.; Savy, P. WO2004/46094 Queen Mary \& Westfield College, University College London, Barts and London NHS Trust, 23.06.03. 
29. Tosatti, P.; Horn, J.; Campbell, A. J.; House, D.; Nelson, A.; Marsden, S. P. Adv. Synth. Catal. 2010, 352, 3153.

30. Hatano, M.; Horibe, T.; Ishihara, K. Angew. Chem. Int. Ed. 2013, 52, 4549.

31. Soucy, F.; Grenier, L. Behnke, M. L.; Destree, A. T.; McCormack, T. A.; Adams, J.; Plamondon, L. J. Am. Chem. Soc. 1999, 121, 9967.

32. Zhong, H. M.; Cohen, J. H.; Abdel-Magid, A. F.; Kenney, B. D.; Maryanoff, C. A.; Shah, R. D.; Villani, Jr., F. J.; Zhang, F.; Zhang, X. Tetrahedron Lett. 1999, 40, 7721.

33. Erichsen, M. N.; Huynh, T. H. V.; Abrahamsen, B.; Bastlund, J. F.; Bundgaard, C.; Monrad, O.; Bekker-Jensen, A.; Nielsen, C. W.; Frydenvang, K.; Jensen, A. A.; Bunch, L. J. Med. Chem. 2010, 53, 7180 .

34. Lutz, A. H.; Schnider, O. Helv. Chim. Acta 1956, 39, 81.

35. Chen, B. L.; Wang, B.; Lin, G. Q. Sci. China Chem. 2014, 57, 945.

36. Bentley, S. A.; Davies, S. G.; Lee, J. A.; Roberts, P. M.; Russell, A. J.; Thomson, J. E.; Toms, S. M. Tetrahedron 2010, 66, 4604.

37. Marin, J.; Didierjean, C.; Aubry, A.; Casimir, J.-R.; Briand, J.-P.; Guichard, G. J. J. Org. Chem. 2004, 69, 130.

38. Davis, F. A.; Chao, B.; Fang, T.; Szewczyk, J. M. Org. Lett. 2000, 2, 1041.

*Corresponding author: E-mail: c.m.marson@ucl.ac.uk FAX:+44(0)20 76797463

\section{Legends}

Figure 1. Representative oxopiperidine derivatives with biological or pharmaceutical activity.

Scheme 1. Synthesis of substituted piperidine-2,4-diones and derivatives via Dieckmann cyclisations. Reagents and conditions: (a) $\mathrm{NH}_{4} \mathrm{OAc}, \mathrm{AcOH}, \mathrm{PhH}$, reflux, 18-72 h; (b) $\mathrm{NaBH}_{4}$ (2.5 equiv.) $\mathrm{AcOH}, 20$ ${ }^{\circ} \mathrm{C}, 3 \mathrm{~h}$; (c) monomethyl malonate, EDC (1 equiv.), $\mathrm{HOBt}\left(1.5\right.$ equiv.), $(i-\mathrm{Pr})_{2} \mathrm{NEt}, \mathrm{CH}_{2} \mathrm{Cl}_{2}, 20{ }^{\circ} \mathrm{C}, 2 \mathrm{~h}$; (d) $\mathrm{NaOMe}$ (2 equiv.), $\mathrm{MeOH}$, reflux, $1 \mathrm{~h}$; (e) $\mathrm{MeCN}, 1 \% \mathrm{H}_{2} \mathrm{O}$, reflux, $1 \mathrm{~h}$.

Scheme 2. Synthesis of $\beta$-keto esters via Weiler dialkylation. Reagents and conditions: (a) $\mathrm{NaH}$ (1.1 equiv.), BuLi (1.1 equiv.), THF, MeI or EtBr (1.1equiv.) EtOH, $20^{\circ} \mathrm{C}, 3 \mathrm{~h}, 97 \%$ (1b), 85\%, (1c); (b) MeI (1.0 equiv.), $\mathrm{K}_{2} \mathrm{CO}_{3}$ (1.5 equiv.), $\mathrm{Me}_{2} \mathrm{CO}, 0^{\circ} \mathrm{C}, 2 \mathrm{~h}, 88 \%$ (1d).

Scheme 3. Synthesis of 2,3-disubstituted piperidine-2,4-diones. Reagents and conditions: (a) Zn (4 equiv.), TMSCl (12 equiv.), $\mathrm{CH}_{2} \mathrm{Cl}_{2}$, THF, reflux, $2 \mathrm{~h}$ then $\mathrm{NaBH}_{4}\left(0.55\right.$ mole equiv.) $\mathrm{EtOH}, 20{ }^{\circ} \mathrm{C}, 3 \mathrm{~h}$, $15 \%$; (b) monomethyl malonate, EDC (1.0 equiv.), $\mathrm{HOBt}\left(1.5\right.$ equiv.), $(i-\mathrm{Pr})_{2} \mathrm{NEt}, \mathrm{CH}_{2} \mathrm{Cl}_{2}, 20{ }^{\circ} \mathrm{C}, 2 \mathrm{~h}$, $67 \%$; (c) $\mathrm{NaOMe}$ (1.3 equiv.), $\mathrm{MeOH}$, reflux, $1 \mathrm{~h}$, then $\mathrm{MeCN}, 1 \% \mathrm{H}_{2} \mathrm{O}$, reflux, $1 \mathrm{~h}, 56 \%$.

Figure 2. Synthesis of substituted piperidine-2,4-diones and derivatives via Dieckmann cyclisations.

Scheme 4. Attempted cyclisation of diester $4 \mathbf{4 f}$. Reagents and conditions: (a) $\mathrm{ClOCCH}_{2} \mathrm{CO}_{2} \mathrm{Me}(1.5$ equiv.), (i-Pr) $)_{2} \mathrm{NEt}\left(4\right.$ equiv.), $0{ }^{\circ} \mathrm{C}$, then $20^{\circ} \mathrm{C}, 1 \mathrm{~h}, 99 \%$; (b) $\mathrm{NaOMe}$ (1.3 equiv.), $\mathrm{MeOH}$, reflux, $1 \mathrm{~h}$, then $\mathrm{MeCN}, 1 \% \mathrm{H}_{2} \mathrm{O}$, reflux, $1 \mathrm{~h}$.

Scheme 5. Enantioselective synthesis of substituted piperidine-2,4-diones and derivatives via Dieckmann cyclisations $(\mathrm{R}=(S)$-1-phenylethyl). Reagents and conditions: (a) BuLi (1 equiv.), THF, $78{ }^{\circ} \mathrm{C}, 30 \mathrm{~min}$; $\mathrm{RCH}=\mathrm{CHCO}_{2} \mathrm{Me}$ (1 equiv.), $-78{ }^{\circ} \mathrm{C}, 30 \mathrm{~min}$; (b) $\left(\mathrm{Ph}_{3} \mathrm{P}\right)_{3} \mathrm{RhCl}(5 \mathrm{~mol} \%)$, aq. $\mathrm{MeCN}$, reflux, $16 \mathrm{~h}$; (c) methyl 3-chloro-3-oxopropanoate (1.1 equiv.), $\mathrm{Et}_{3} \mathrm{~N}\left(1.2\right.$ equiv.), $0{ }^{\circ} \mathrm{C}$ to $20{ }^{\circ} \mathrm{C}, 1 \mathrm{~h}$; (d) $\mathrm{NaOMe}(1.1$ equiv.), reflux $1 \mathrm{~h}$, then dil. $\mathrm{HCl}$; (e) $1 \%$ water in $\mathrm{MeCN}$, reflux $1 \mathrm{~h}$; (f) $\mathrm{MsOH}(0.9$ equiv.), toluene, reflux, $3 \mathrm{~h}$.

Figure 3. Enantioselective synthesis of substituted piperidine-2,4-diones and derivatives via Dieckmann cyclisations $(\mathrm{R}=(S)$-1-phenylethyl). 